\title{
Unique Testing Capabilities of the NASA Langley Transonic Dynamics Tunnel, an Exercise in Aeroelastic Scaling
}

\author{
Thomas G. Ivanco* \\ Aeroelasticity Branch, NASA Langley Research Center, Hampton, VA 23681
}

\begin{abstract}
The NASA Langley Research Center Transonic Dynamics Tunnel (TDT) is well known for its aeroelastic testing capabilities. Its large size, transonic speed range, variable pressure capability, and use of either air or R-134a heavy gas as a test medium enable unparalleled manipulation of flow-and-structure-dependent scaling quantities. This paper will present select scaling quantities that are important for the proper characterization of any dynamic phenomenon and many static aeroelastic phenomena. An analytical demonstration is presented comparing the unique ability of TDT to match these scaling quantities by comparison to other leading facilities with other test mediums. Complementary to aeroelastic testing, the TDT is well-suited for high risk testing and for those tests that require unusual model mount or support systems. Examples are presented. In addition to its unique aeroelastic testing capabilities, flow quality and Reynolds number capability are briefly discussed. Finally, the ability of the TDT to support future NASA research thrusts and likely vehicle designs is presented.
\end{abstract}

\section{Nomenclature}

$a$

$a_{e}$

$b$

$C_{L}$

$C_{Q}$

$E$

$f$

speed of sound, $\mathrm{ft} / \mathrm{s}$

$=$ distance from the mid-chord to the elastic axis, normalized with semi-chord, + aft

$=$ airfoil semi-chord, $\mathrm{ft}$

$=$ lift coefficient

$=$ torque coefficient, rotorcraft

$=$ Young's modulus of elasticity, lbs/in ${ }^{2}$

$=$ frequency, $\mathrm{Hz}$

$=$ acceleration due to gravity, $\mathrm{ft} / \mathrm{s}^{2}$

$=$ area moment of inertia, in ${ }^{4}$ or $\mathrm{ft}^{4}$

$=$ reduced frequency

$=$ characteristic length, in or $\mathrm{ft}$

$=$ mass per unit length, slugs $/ \mathrm{ft}$

= Mach number

$=$ Bending moment about the neutral axis, in-lbs

$=$ dynamic pressure, $\mathrm{lbs} / \mathrm{ft}^{2}$

$=$ radius of gyration

$=$ Reynolds number

$=$ reference area, $\mathrm{ft}^{2}$ (such as wing area or cross-sectional area of test section)

$=$ reduced velocity

$=$ velocity, $\mathrm{ft} / \mathrm{s}$

$=$ Static unbalance; distance from C.G. to elastic axis normalized with semi-chord, + aft

$=$ perpendicular distance to the neutral axis (for bending stress analysis), in

$=$ ratio of specific heats $\left(C_{P} / C_{V}\right)$

$=$ scale factor, model-scale to full-scale ratio of variable

$=$ fluid viscosity, $\mathrm{lb}-\mathrm{sec} / \mathrm{ft}^{2}$

$=$ mass ratio

$=$ fluid density, slug/ $\mathrm{ft}^{3}$

$=$ material density, slug/ $\mathrm{ft}^{3}$

${ }^{*}$ Research Aerospace Engineer, Aeroelasticity Branch, Mail Stop 340, AIAA Senior Member. 


$\begin{array}{ll}\sigma & =\text { solidity, ratio of blade area to rotor disk area } \\ \sigma_{\text {stress }} & =\text { bending stress, lbs/in }{ }^{2} \\ \omega & =\text { circular frequency }(=2 \pi f), \mathrm{rad} / \mathrm{s} \\ \text { subscripts } & \\ F & =\text { flutter } \\ h & =\text { plunge } \\ \alpha & =\text { pitch }\end{array}$

\section{Introduction}

$\mathrm{T}$

HE NASA Langley Research Center (LaRC) Transonic Dynamics Tunnel (TDT) has been operational since 1960 investigating a wide range of aeroelastic and non-aeroelastic phenomena. ${ }^{1-4}$ A dedicated aeroelastic test facility, the TDT is a large, variable pressure, transonic wind tunnel that can use either air or heavy gas (R-134a) as a test medium. Unlike typical wind tunnel testing which focuses upon modeling the aerodynamics experienced by a flight vehicle, the study of aeroelasticity requires modeling of the structural response of the flight vehicle when exposed to representative aerodynamics. The coupling between fluid and structural interaction can be described by a series of non-dimensional scaling parameters. Fluid and structural-response coupling tends to increase in sensitivity as the sonic condition is approached since the lift-curve-slope steepens as a function of Mach number and slight structural perturbations have an increasing aerodynamic effect. ${ }^{5}$ As a result, many vehicles experience a "transonicdip" in the stability boundary with the lowest stability margins existing at high subsonic Mach numbers. ${ }^{5,6}$ High subsonic Mach numbers are where most transport aircraft cruise, where high performance aircraft pass through, where launch vehicles experience the highest unsteady buffet loads during ascent, ${ }^{7}$ and are also where computational fluid dynamics (CFD) codes have the greatest difficulty in modeling flow physics; especially unsteady flow physics. ${ }^{8}$ Therefore, experimental data in the transonic speed range is of particular importance. Typically regarded as the world's premier aeroelastic test facility, ${ }^{9-13}$ TDT fulfills a unique niche in the wind tunnel infrastructure as a result of its unparalleled ability to manipulate fluid-structure scaling parameters.

This paper will briefly describe TDT features and the general characteristics of other leading transonic facilities. This will be followed by a systematic evaluation of aeroelastic scaling parameters and their importance upon different classes of wind tunnel tests. This paper will then quantify the unique capability of TDT to match flightscaled quantities, and will recognize complementary safety features and support systems. Additionally, a preliminary, but promising, review of TDT flow quality and Reynolds number capability has been conducted; however, the details of this discussion are reserved for a future publication. Finally, the likely use of TDT to support future aircraft designs is discussed.

\section{Wind Tunnel Facilities}

\section{A. NASA TDT}

The TDT is a continuous-flow, single-return, slotted-test-section wind tunnel capable of testing at total pressures from 0.005 to 1.0 atmospheres, over a Mach number range from near zero to 1.20. The test section of the TDT is 16 by 16 feet square with cropped corners yielding a cross-sectional area of 248 square feet. A sketch of the TDT is shown in Fig. 1 with cutaways revealing some key features of the wind tunnel. Various model support systems are available offering a wide range of options to enable proper simulation of many dynamic and static phenomena. Models can be mounted with: various sting mounts; various sidewall mounts; a floor-mounted turntable; a twocable, free-flight support system; a helicopter test apparatus; a tilt-rotor test apparatus; or custom mount systems. ${ }^{1-}$

${ }^{4,14}$ In addition to the mount type, such as sting or sidewall, various forms of model actuation are also available. The sidewall turntable can either be electrically actuated, or hydraulically actuated with a high-frequency system known as the oscillating turntable (OTT). Some tests have also utilized a forced oscillation and/or free-to-pitch cross-bar support in conjunction with the OTT to acquire dynamic stability derivatives. Similarly, the sting can be remotely operated in pitch and roll. Some tests have also used a free-to-roll sting mount or dynamic balance installed on the sting that is capable of oscillating the model to acquire dynamic stability derivatives in pitch or yaw degrees of freedom. Examples of custom mount systems include a two degree of freedom sidewall support enabling pitch and plunge motion for a semi-span model; and a centerline pole enabling a pitch and plunge motion of a full-span model. Both of these custom examples closely simulated free-flight models and were developed to investigate active control 
technologies that required integration with primary flight controls. Fig. 2 illustrates various model mount systems and unique models tested at TDT, and Refs. 1-4 and 14 provide additional details.

Supplementing the model mount systems are several auxiliary tunnel systems to facilitate model operations including: high pressure air or R-134a; motor-generator sets to provide conditioned electrical power; hydraulic systems for model actuation; a chilled water cooler; and an air stream oscillation system for gust response studies or model excitation.

To enable high risk testing often associated with dynamic investigations, the TDT possesses unique safety features such as a series of bypass valves that rapidly reduce testsection flow velocity, a model catch screen to protect the drive fan in the event of a model failure, and a wall of observation windows enabling a direct model view from the control room during operation. TDT also utilizes a dedicated dynamic data system providing realtime displays of various analog and/or digital signals or computed quantities. These real time displays can be used to monitor not only model loads, but also to foresee the onset of an aeroelastic instability through the use of dynamic signal analysis tools. A synchronous time history of all signals is acquired for each

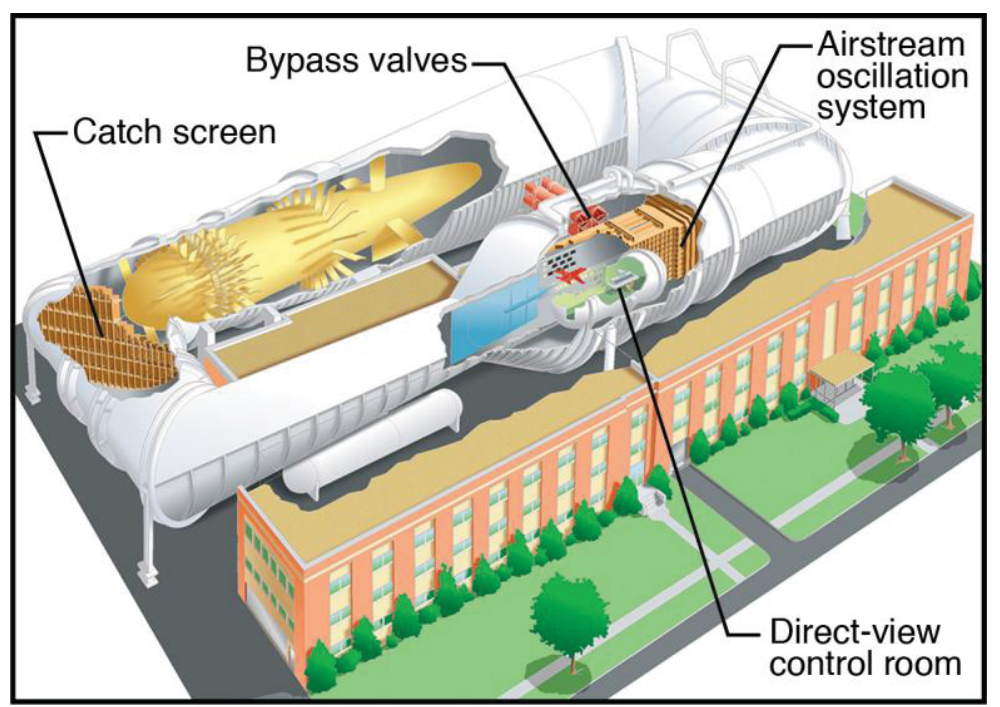

Figure 1. Sketch of the Transonic Dynamics Tunnel with cutaways revealing key features.

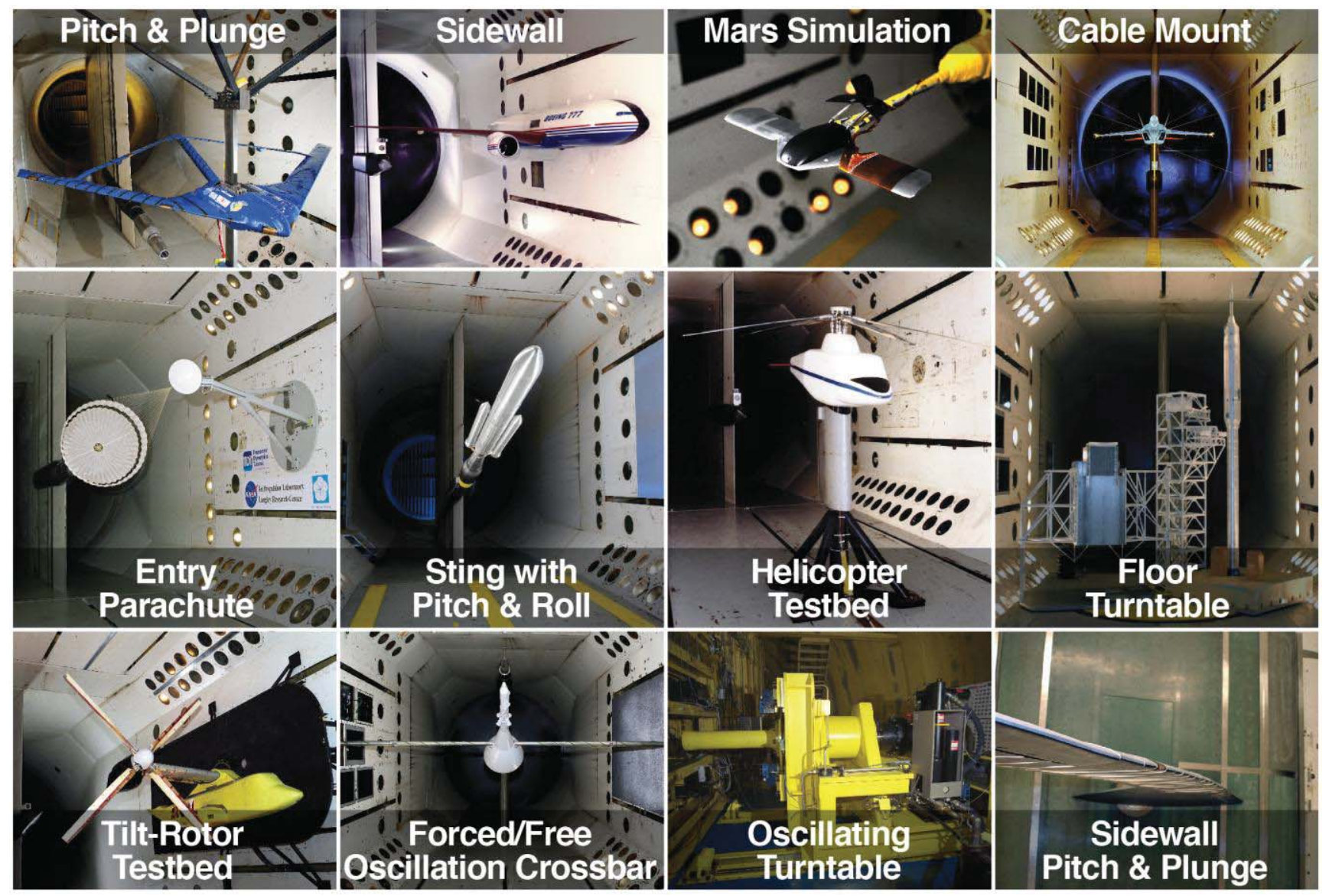

Figure 2. TDT Model mount systems and unique models.

3

American Institute of Aeronautics and Astronautics 
data point. A new data system is under development for use at TDT that will have an increased scan rate, adaptability for an increased channel count, and an increased reliability. More details regarding the calculation of tunnel parameters can found in Ref. 15.

Finally, co-located with the TDT facility is the NASA Aeroelasticity Branch. The Aeroelasticity Branch is comprised of a team of researchers who study various forms of aeroelasticity with computational and experimental methods. The research branch often facilitates TDT tests and can offer unique expertise and test guidance.

\section{B. Other Wind Tunnel Facilities}

In order to quantify the capabilities of TDT within the wind tunnel infrastructure, a brief description of other wind tunnel facilities is required. Since they satisfy a different realm of testing, subsonic, supersonic, and hypersonic facilities are neglected with the exception of the San Diego Air and Space Museum Low Speed Wind Tunnel (LSWT). The LSWT possesses a rapid flow reduction capability and is frequently used for aeroelastic testing. Therefore, attention to LSWT is warranted even though it is a subsonic facility. Table 1 is a list of all wind tunnel facilities used for comparison in this document and includes all of the leading and currently operational transonic wind tunnels known to the author at the time of publication. Included in this table are the test section size, Mach number capabilities, test mediums used, maximum Reynolds number capabilities, and the maximum dynamic pressure that is reached at the maximum Reynolds number. Particular attention should be paid to LSWT, S2MA, and T-128 in relation to TDT since these wind tunnels frequently conduct aeroelastic tests. Transonic wind tunnels also exist in Singapore, Taiwan, South Africa, Israel, Japan, and are believed to exist in China in addition to some universities. All of these wind tunnels utilize air and are similar in size or smaller than the AeroSystems Engineering (formerly Fluidyne) Channel 10 transonic wind tunnel, and have less pressure capability than the STARCS T1500. It should also be noted that heavy gas has been used as a test medium in other facilities such as the 0.3-meter Transonic Cryogenic Tunnel and the 20-inch Mach-6 CF4 Tunnel. ${ }^{10,16}$ However, both of these facilities are substantially smaller than TDT and do not offer the same capability.

Maximum dynamic pressure values were calculated for some wind tunnels (denoted by an *) based upon the available information published by the owning organization. Some facilities publish the maximum total pressure and the corresponding dynamic pressure was derived by using compressible isentropic relations, the equation of state, and the Mach number where maximum total pressure occurs. Additionally, Reynolds number was calculated using $0.1 \sqrt{S}$ as the characteristic length, where $S$ is the cross-sectional area of the test section. 
Table 1. Wind Tunnel Facilities

\begin{tabular}{|c|c|c|c|c|c|c|c|c|}
\hline Organization & Name & Country & Mach Range & $\begin{array}{c}\text { Test } \\
\text { Section } \\
\text { Size (ft) }\end{array}$ & $\begin{array}{c}\text { Test } \\
\text { Medium }\end{array}$ & $\begin{array}{l}\text { Variable } \\
\text { Pressure }\end{array}$ & $\begin{array}{c}\text { Max } \\
\text { Reynolds } \\
\text { Number } \\
(0.1 \sqrt{S})\end{array}$ & $\begin{array}{c}\text { Max } \\
\text { Dynamic } \\
\text { Pressure } \\
\text { (psf) }\end{array}$ \\
\hline NASA LaRC & NTF & USA & $0.2-1.2$ & $8.2 \times 8.2$ & $\begin{array}{l}\text { Cryo } \mathrm{N}_{2} \\
\text { Air }\end{array}$ & yes & $\begin{array}{l}118.5 \times 10^{6} \\
18.5 \times 10^{6}\end{array}$ & $\begin{array}{l}5,500 * \\
5,500 *\end{array}$ \\
\hline ETW & ETW & Germany & $0.15-1.35$ & $6.56 \times 7.87$ & $\begin{array}{l}\text { Cyro } \mathrm{N}_{2} \\
\text { Air }\end{array}$ & yes & $50.4 \times \underset{\S}{x} 10^{6}$ & $\underset{\S}{3,500 *}$ \\
\hline NASA LaRC & TDT & USA & $0.05-1.2$ & $16 \times 16$ & $\begin{array}{c}\text { R-134a } \\
\text { Air }\end{array}$ & yes & $\begin{array}{l}15.3 \times 10^{6} \\
4.72 \times 10^{6}\end{array}$ & $\begin{array}{l}550 \\
320\end{array}$ \\
\hline DNW & KRG & Germany & $0.3-0.95$ & $1.31 \times 1.15$ & Cyro $\mathrm{N}_{2}$ & yes & $15.0 \times 10^{6}$ & $7,480^{*}$ \\
\hline STARCS & $\mathrm{T} 1500$ & Sweden & $0.19-1.2$ & $5 \times 5$ & Air & yes & $12.0 \times 10^{6}$ & $3,114^{*}$ \\
\hline AEDC & $16 \mathrm{~T}$ & USA & $0.06-1.6$ & $16 \times 16$ & Air & yes & $11.6 \times 10^{6}$ & 1,161 \\
\hline TsAGI & T-128 & Russia & $0.15-1.7$ & $9.02 \times 9.02$ & Air & yes & $11.3 \times 10^{6}$ & 1,671 \\
\hline NASA ARC & $11 \mathrm{ft}$ & USA & $0.2-1.5$ & $11 \times 11$ & Air & yes & $10.6 \times 10^{6}$ & 1,900 \\
\hline NASA LaRC & 0.3 meter cyro & USA & $0.1-0.95$ & $1.08 \times 1.08$ & $\begin{array}{l}\text { Cryo } \mathrm{N}_{2} \\
\text { Air }\end{array}$ & yes & $10.0 \times \underset{\S}{\times} 10^{6}$ & $\underset{\S}{5,687}$ \\
\hline DNW & HST & Germany & $0.15-1.35$ & $6.56 \times 5.91$ & Air & yes & $9.0 \times 10^{6}$ & $2,040^{*}$ \\
\hline Calspan & TWT & USA & $0-1.3$ & $8 \times 8$ & Air & yes & $8.4 \times 10^{6}$ & 2,600 \\
\hline TsAGI & T-106 & Russia & $0.15-1.1$ & $\begin{array}{c}8.14 \\
\text { diameter } \\
\end{array}$ & Air & yes & $7.7 \times 10^{6}$ & 1,211 \\
\hline ONERA & S1MA & France & $0.05-1.0$ & $\begin{array}{c}25.4 \\
\text { diameter }^{\dagger}\end{array}$ & Air & no & $7.5 \times 10^{6}$ & $668 *$ \\
\hline Lockheed Martin & $\begin{array}{c}\text { HSWT } \\
\text { w/ trans. nozzle }\end{array}$ & USA & $0.3-1.8$ & $4 \times 4$ & Air & yes & $6.0 \times 10^{6}$ & 3,200 \\
\hline ONERA & $\begin{array}{c}\text { S2MA } \\
\text { w/trans. nozzle }\end{array}$ & France & $0.1-1.3$ & $5.81 \times 5.74$ & Air & yes & $5.3 \times 10^{6}$ & $1,550 *$ \\
\hline $\begin{array}{l}\text { Aircraft Research } \\
\text { Association }\end{array}$ & $\begin{array}{c}\text { Transonic Wind } \\
\text { Tunnel }\end{array}$ & UK & $0.2-1.4$ & $8 \times 12$ & Air & yes & $4.5 \times 10^{6}$ & $1,055^{*}$ \\
\hline Boeing & BTWT & USA & $0.3-1.1$ & $8 \times 12$ & Air & no & $4.1 \times 10^{6}$ & 840 \\
\hline NASA GRC & $\begin{array}{c}8 \mathrm{x} 6 \mathrm{ft} \\
\text { supersonic }\end{array}$ & USA & $0.25-2.0$ & $8 \times 6$ & Air & yes & $3.33 \times 10^{6}$ & 1,340 \\
\hline $\begin{array}{l}\text { AeroSystems } \\
\text { Engineering }\end{array}$ & $\begin{array}{c}\text { Channel } 10 \\
\text { Trans. Wind } \\
\text { Tun. }\end{array}$ & USA & $0.13-1.5$ & $5.5 \times 5.5$ & Air & no & $2.31 \times 10^{6}$ & $944 *$ \\
\hline DNW & TWG & Germany & $0.3-2.2$ & $3.28 \times 3.28$ & Air & yes & $1.8 \times 10^{6}$ & $1,219 *$ \\
\hline AMRL & $\begin{array}{c}\text { Transonic Wind } \\
\text { Tunnel } \\
\end{array}$ & Australia & $0-1.2$ & $2.65 \times 2.65$ & Air & yes & $0.19 \times 10^{6}$ & $1,627^{*}$ \\
\hline Boeing & $\begin{array}{c}\text { PSWT } \\
\text { w/trans. nozzle }\end{array}$ & USA & $0.45-1.6$ & $4 \times 4$ & Air & yes & $\$$ & * \\
\hline $\begin{array}{l}\text { National } \\
\text { Research Council }\end{array}$ & $\begin{array}{l}\text { 1.5m Trisonic } \\
\text { Blowdown } \\
\text { Wind Tunnel }\end{array}$ & Canada & $0.1-4.25$ & $5 \times 5$ & Air & yes & $\begin{array}{c}\text { not } \\
\text { published }\end{array}$ & $\begin{array}{c}\text { not } \\
\text { published }\end{array}$ \\
\hline $\begin{array}{l}\text { San Diego Air } \\
\text { and Space } \\
\text { Museum }\end{array}$ & LSWT & USA & $0.04-0.36$ & $8 \times 12$ & Air & no & $2.4 \times 10^{6}$ & 190 \\
\hline
\end{tabular}

*Maximum dynamic pressure estimated by using published total pressure, compressible isentropic relations, and equation of state

${ }^{\dagger}$ S1MA has an 8 meter shell with various test section inserts offering a range of cross sectional areas from $414.4 \mathrm{ft}^{2}$ to $505.9 \mathrm{ft}^{2}$

"not published for the transonic nozzle

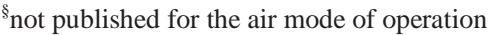

\section{Aeroelastic Scaling and Parameter Relevance for Different Classes of Tests}

\section{A. Typical Aeroelastic Scaling Parameters}

Model similitude to a full-scale vehicle requires that wind-tunnel flow conditions and model structural characteristics satisfy various relationships. ${ }^{1,5,17-20}$ Some facilities study the aeroelastic and buffet phenomena of a wind tunnel model and tunnel support system without relation to a flight vehicle. ${ }^{21}$ An important distinction for TDT 
is that the aeroelastic and buffet phenomena discussed in this document refer to events experienced by a flight vehicle and the corresponding ability of a wind tunnel model to simulate those events. This distinction is of the utmost importance.

Important parameters for aerodynamic scaling are: a relation of velocity to speed of sound (Mach number); and a relation of the fluid momentum to viscous forces (Reynolds number). An additional parameter for static aeroelastic scaling is: a relation of the elastic forces to the aerodynamic forces (elastic scaling). Two additional parameters that are important for dynamic aeroelastic scaling are: a relation of the speed of structural oscillations to the speed of the passing fluid (reduced frequency); and a relation of the structural mass to fluid mass (mass ratio). For special cases of dynamic aeroelastic scaling, a relation of the aerodynamic pressure forces to gravitational forces (Froude number) may also be important. Finally, even though they are not listed here as scaling parameters, the mode shapes of vibration, and the aircraft geometry are also important similitude properties.

Equations for the aeroelastic scaling parameters are presented in Eq. (1) through Eq. (6) for conditions where gas thermodynamic scaling can be neglected (Refs. 1, 5, 17-20). The application and importance of these parameters will be discussed in the following sections.

$$
\begin{gathered}
\text { Mach Number }(M)=\frac{V}{a} \\
\text { Reynolds number }(R e)=\frac{\rho V L}{\mu} \\
\text { elastic scaling }(\varepsilon)=\frac{E I}{\rho V^{2} L^{4}} \\
\text { reduced frequency }(k)=\frac{\omega L}{V} \\
\text { mass ratio }\left(\mu_{m}\right)=\frac{m}{\pi \rho b^{2}}{ }^{\dagger} \\
\text { Froude number }(F r)=\frac{V}{\sqrt{g L}}
\end{gathered}
$$

\section{B. Scaling Parameter Importance for Different Classes of Wind Tunnel Tests}

Matching all scaling parameters between a flight vehicle and sub-scale model is not possible and often unnecessary. The objectives of the test and available resources need to be evaluated during model and experiment design. With regard to scaling parameter importance, aeroelastic tests can be summarized as either static or dynamic. Static aeroelastic tests study phenomena such as divergence or control surface reversal that result from steady elastic deformation of the structure due to applied aerodynamic loads. Dynamic aeroelastic tests study phenomena such as flutter; gust load alleviation; ride quality; aeroservoelasticity; buffet, with dynamically-scaled models; and launch vehicle ground wind loads. In the study of dynamic aeroelasticity, inertial properties, mode shapes and frequencies dictate response behavior in addition to structural deflection and aerodynamic loads. Rotorcraft tests are considered a separate class of test since Froude number, in addition to other dynamic parameters, also plays a critical role relating the amount of blade deflection for a given aerodynamic load and rotor speed., ${ }^{1,19,20}$ Rigid buffet tests, conducted with an essentially rigid model, are classified here as a special case of aerodynamic performance tests. Since neither the deflection nor dynamics of the vehicle are simulated in rigid buffet tests, they are not aeroelastic in nature. However, reduced frequency still dictates the fluctuating pressure behavior and heavy gas offers scaling advantages that drive transducer frequency requirements. Also, the need to acquire synchronized high frequency data, often in the aeroacoustic bandwidth, across many transducers requires specialized data systems. Table 2 is a summary of the different classes of wind tunnel tests and a qualitative description of scaling parameter importance.

\footnotetext{
${ }^{\dagger}$ Mass per unit length $(m)$ includes structural weight and non-structural weight such as ballast
} 
The requirement to match Mach number is well understood and is critical for tests where compressibility effects dominate the aerodynamics. Similarly, the importance of Reynolds number is well understood and critical when boundary layer interactions, drag, or flow separation is of primary concern. Otherwise, it may adequately satisfy the test requirements to test in the same Reynolds number regime such that the boundary layer is turbulent, or to apply artificial roughness to prematurely trip the boundary layer to facilitate scaling to flight Reynolds numbers. Additionally, any fixed-wing model that enforces similitude of reduced frequency and mass ratio will automatically satisfy elastic scaling since the frequency of a structure is fixed for a defined geometry, weight, and elasticity. In contrast, rotor system dynamic frequencies are dominated by rotor speed and require additional attention to simultaneously match blade elasticity and rotor system dynamics. Froude number scaling is important in the investigation of fixed-wing dynamics when the gravitational forces are not negligible. It is also desired, although not critical, for any free-flight models as this dictates the $1 \mathrm{~g}$ trim angle of attack, otherwise it is typically negligible for fixed-wing tests. The importance of the remaining scaling parameters requires additional explanation, and is highlighted in the following examples.

Table 2. Aeroelastic Scaling Parameters, Relative Importance

\begin{tabular}{|c|c|c|c|c|c|}
\hline \multirow[b]{2}{*}{$\begin{array}{c}\text { Scaling } \\
\text { Parameter }\end{array}$} & \multicolumn{5}{|c|}{ Wind Tunnel Test Class } \\
\hline & $\begin{array}{l}\text { Aerodynamic } \\
\text { Performance }\end{array}$ & $\begin{array}{c}\text { Static } \\
\text { Aeroelasticity }\end{array}$ & $\begin{array}{c}\text { Dynamic } \\
\text { Aeroelasticity }\end{array}$ & $\begin{array}{c}\text { Rotorcraft } \\
\text { Performance }\end{array}$ & $\begin{array}{l}\text { Rotorcraft } \\
\text { Stability }^{\dagger}\end{array}$ \\
\hline $\begin{array}{l}\text { Mach } \\
\text { Number }\end{array}$ & critical & critical & critical & critical & critical $^{*}$ \\
\hline $\begin{array}{l}\text { Reynolds } \\
\text { Number }\end{array}$ & critical & preferred & preferred & preferred & preferred \\
\hline $\begin{array}{l}\text { Elastic } \\
\text { Scale }\end{array}$ & N/A* & critical & critical & critical & critical \\
\hline $\begin{array}{l}\text { Reduced } \\
\text { Frequency }\end{array}$ & N/A & N/A & critical & critical & critical \\
\hline Mass Ratio & N/A & N/A & critical & critical & critical \\
\hline $\begin{array}{l}\text { Froude } \\
\text { Number }\end{array}$ & N/A & N/A & $\begin{array}{l}\text { preferred for } \\
\text { free-flight or } \\
\text { free-to-roll }\end{array}$ & critical $^{*}$ & critical \\
\hline
\end{tabular}

*N/A denotes "not applicable" as a scaling parameter for the specified class of wind tunnel test

†For this paper, "rotorcraft stability" refers to the aeroelastic stability and not rigid-body stability and control

¥This scaling parameter is not typically matched in conventional wind tunnel testing even though it is of critical importance

1) Theoretical Example of Dynamic Scaling Importance for Flutter Boundary

Reduced frequency and mass ratio typically define dynamic scaling. ${ }^{20}$ To analyze the effect of reduced frequency, consider an airfoil that is impulsively pitched from a high angle of attack to zero degrees angle of attack. There will be a vortex shed from the leading edge that will considerably affect the pressure distribution on the airfoil. The time history of lift and pitching moment following the impulsive motion are dependent upon the time it takes the flow to transport this vortex off of the airfoil surface and far downstream. As the transport time (flow velocity) is changed, the time history of the aerodynamic forces follows accordingly. Similarly, if one now considers an airfoil continuously oscillating in pitch, the phase between lift, moment, and pitch angle is related to the ratio of flow speed to airfoil size and oscillation frequency. An analogy of this effect in the field of structural dynamics is the resonant excitation of a lightly-damped pendulum. As the phase relationship between position and force changes, the force could either increase or decrease the amplitude." If the phasing relationship is not matched in two otherwise identical systems, one could derive opposite trends regarding force magnitude and response amplitude. In

\footnotetext{
* This physical example should not be confused with the free response of a structure subject to an independent force. This physical example is one such that the excitation force is a function of the current structural position, analogous to aeroelasticity.
} 
the field of aeroelasticity, this phase relationship is captured in the reduced frequency $(k)$ scaling parameter, and it is therefore one of the most important scaling parameters characterizing dynamic aeroelasticity.

Altering mass ratio has a different effect upon the dynamic response. In the study of Newtonian physics, force is equal to mass times acceleration, and as the mass changes so does the magnitude of acceleration for a fixed force. This manifests itself in aeroelasticity by affecting the magnitude of oscillation, and it could also alter the manner in which the modes interact. In the presence of structural and aerodynamic damping, an incorrectly scaled mass ratio has the potential to mask an otherwise unstable or excite an otherwise stable mode interaction.

To quantify the importance of the reduced frequency and mass ratio scaling parameters, a theoretical study is presented here investigating the flutter boundary of a two-dimensional airfoil of unit span with pitch and plunge degrees of freedom. In order to capture the effect of reduced frequency, an aerodynamic theory that captures the aerodynamic-structural phase relationship of an oscillating airfoil is required. Theodore Theodorsen developed such a theory, known as Theodorsen Aerodynamics, and it has been used extensively in the field of aeroelasticity. ${ }^{5,6,23}$ The phase parameters within Theodorsen Aerodynamics are a direct function of reduced frequency which enables convenient study of its effects in addition to other variables. The equations used were cast as shown by Ref. 6, and the results were validated by comparison to the results shown in Ref. 6 .

The two degree of freedom (2-DOF) pitch and plunge airfoil represents the classic bending-torsion flutter scenario. Following the convention of Ref. 6, the flutter velocity of this system is a function of five independent flutter parameters. These parameters are:

1. Mass ratio $\left(\mu_{m}\right)$; see Eq. (5)

2. Radius of gyration $\left(r_{o}\right)$; a relationship of mass to the mass moment of inertia

3. Static unbalance $\left(X_{o}\right)$; distance from C.G. to elastic axis normalized with semi-chord, + aft

4. Frequency ratio of plunge-torsion natural frequencies in a vacuum $\left(\omega_{h} / \omega_{\alpha}\right)$

5. Location of the elastic axis $\left(a_{e}\right)$; distance from mid-chord normalized with semi-chord, + aft

Ref. 6 normalizes the flutter velocity $\left(V_{F}\right)$ with semi-chord and pitch natural frequency in a vacuum $\left(\omega_{\alpha}\right)$ as shown in Eq. (7).

$$
U_{F}=V_{F} / b \omega_{\alpha}
$$

By normalizing flutter velocity in this fashion, the reduced flutter velocity $\left(U_{F}\right)$ is then independent of airfoil size or other characteristics and is only a function of the five independent flutter parameters. Reduced frequency is not listed here as one of the independent flutter parameters; however, it is embedded in the Theodorsen aerodynamics. Additionally, this theory neglects compressibility or Reynolds number effects and dimensional velocity is therefore unimportant.

A series of flutter solutions were acquired for various values of the flutter parameters. Select results for values of $\mu_{m}=20$, and $r_{\alpha}{ }^{2}=0.25$ are presented as a function of frequency ratio $\left(\omega_{h} / \omega_{\alpha}\right)$ in Fig. 3a and Fig. 3b with values of $X_{o}$ and $a_{e}$ as labeled for each plot. Nominal results are denoted "Dynamically-scaled (R-134a)." Also included in Fig. 3 are results for an otherwise identical analysis where $\mu_{m}$ is increased by a factor of 4.59 , and reduced frequency is reduced by a factor of 2.14 within the aerodynamic equations. These modified results are denoted as "Mis-Scaled (air)" in Fig. 3a and 3b. The factors of 4.59 and 2.14 are not arbitrarily chosen. A model that is dynamically-scaled for testing in R-134a will have 4.59 times too much mass and 2.14 times too low a frequency for testing in air at otherwise identical conditions. At this point, it is only assumed that the system in question is dynamically scaled for R-134a. It will be demonstrated in later sections of this paper that dynamically scaling a model for air testing is difficult, and the relation represented in Fig. 3 is a likely outcome.

The mis-scaled flutter predictions presented in Fig. 3 not only occur at a significantly different reduced velocity, but they also follow a different trend with regard to a change in frequency ratio. Recall that these solutions are calculated for a simple 2-DOF system, with no structural damping, where the flutter mechanism is fixed as a bending-torsion coupling, and compressibility is neglected. An actual flight vehicle is substantially more complex and contains an infinite number of modes that may interact in various ways. Changing reduced frequency and mass ratio of an actual vehicle is likely to have more significant effects. Therefore, testing a model that is not dynamically-scaled could result in trends that are opposite to actual behavior. This casts doubt upon parameter variation testing even when done about a known flutter point unless the model is dynamically-scaled. 


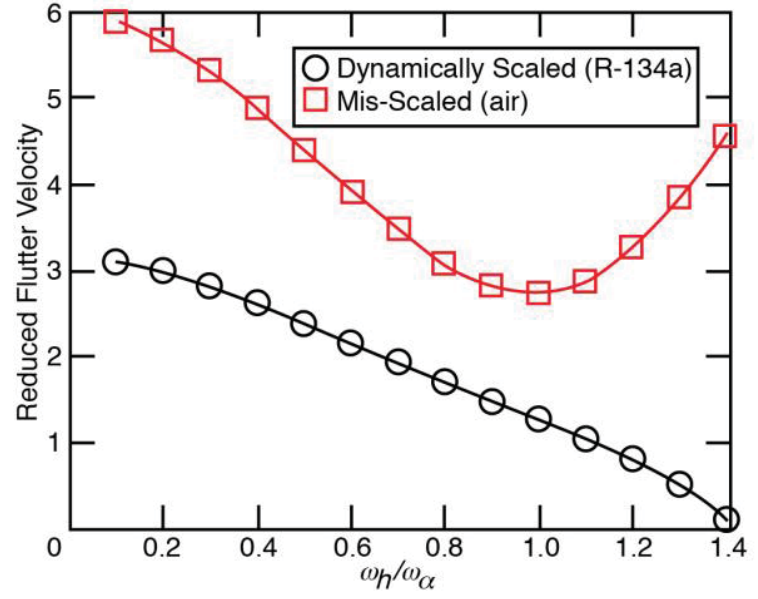

a) $X_{o}=0.15, a_{e}=-0.4$

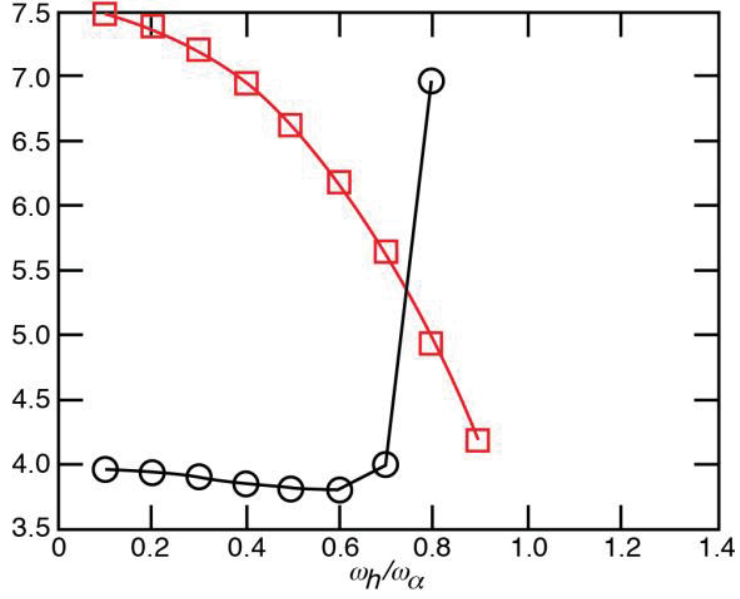

b) $X_{o}=0.0, a_{e}=-0.35$

Figure 3. Flutter boundary, 2-DOF airfoil with Theodorsen aerodynamics, $\mu_{m}=20$, and $r_{\alpha}{ }^{2}=0.25$.

2) Experimental Example of Dynamic Scaling Importance for Flutter Boundary

In the fall of 2010, a knowledge capture effort was conducted at TDT to train personnel in the operation of cablemounted models. Because of availability, the F-16 cable-mounted flutter model was used. This model is dynamically-scaled for testing in R-134a and was tested an extensive amount in TDT in previous efforts. ${ }^{2}$ The most stable model configuration that offered the greatest stability boundary was chosen for the training effort. To simplify the test and save on resources, it was decided to use air as a test medium in lieu of R-134a. However, during the air test, the model encountered a flutter point that was a combination of second-wing-bending and wing-tip-torsion. This flutter instability did not exist in previous or subsequent R-134a testing, and does not exist on the actual flight vehicle. It was concluded by the test team that the existence of this new flutter instability encountered in air was a direct result of the model not being dynamically scaled since the same model and test setup was flown at otherwise identical conditions.

3) Experimental Example of Dynamic Scaling Importance for Rotorcraft Performance

Several characteristics make sub-scale model simulation of rotorcraft difficult. The aerodynamics are transonic and highly unsteady, and by design the primary control forces produced by the rotor are governed by blade dynamics. In addition to matching the normal dynamic scaling parameters $\left(k\right.$ and $\left.\mu_{m}\right)$, matching Froude number also becomes important. It has generally been accepted that simultaneously matching Froude number and Mach number is not typically possible, whereas matching mass ratio, stiffness, and reduced frequency is mandatory. ${ }^{1,19,20}$ Ref. 20 recommends matching Mach number for rotorcraft performance tests and matching Froude number for rotorcraft stability tests. It should be noted that in the study of rotorcraft, mass ratio is characterized by the non-dimensional parameter of Lock number. Satisfying Eq. (5) in the current paper has the same effect as matching Lock number. Similarly, in the study of rotorcraft, reduced frequency is satisfied by matching advance ratio, the ratio of forward speed to hover tip speed. For consistency, this paper will continue to use mass ratio and reduced frequency as the characteristic parameters.

Secondary in importance to Mach number for rotorcraft performance testing, ${ }^{20}$ Froude number still has a significant effect on performance data. Froude number directly relates the aerodynamic forces to blade weight. Blade weight is directly related to the magnitude of rotational-inertia forces, commonly called centrifugal force. Since the aerodynamic loads of a rotor blade are reacted primarily by centrifugal force, Froude number then characterizes the balance between aerodynamic, gravitational, and rotational-inertia forces that dictate the degree of blade flapping for otherwise dynamically-scaled rotors. Even for a steady condition of constant rotor thrust and constant control input; varying Froude number will vary the degree of rotor disk coning and disk tilt. The degree of coning will affect the angle of attack distribution along the span of the rotor blade. Therefore, not matching Froude number for rotorcraft performance testing is analogous to not matching wing twist in a fixed-wing test. 
Early in the history of TDT, a rotorcraft performance test was conducted comparing the sub-scale heavy gas data acquired in $\mathrm{TDT}^{\S}$ with full-scale air data acquired in the NASA Full-scale Aerodynamic Complex (NFAC) for an AH-1G rotor. ${ }^{1,19,24}$ The TDT test studied variations in Reynolds number and dynamic scaling. Due to the use of heavy gas in TDT, the dynamically-scaled model was able to simultaneously match Mach number and Froude number in addition to elastic scaling, mass ratio and reduced frequency. Full-scale Reynolds number was achieved by using rotor blades with a larger chord and neglecting dynamic scaling. A summary of the data acquired is shown in Fig. 4, courtesy of Ref. 1 , where $C_{L}$ is the lift coefficient and $C_{Q}$ is the torque coefficient. In Fig. 4, $\sigma$ denotes solidity defined as the ratio of blade area to rotor disk area. Model configurations where $\sigma$ does not equal the full-scale value indicate those configurations with larger chord that are also not dynamically-scaled.

Reynolds number variation does affect the data; however, the Reynolds number effect is negligible by comparison to the effect of changing dynamic scaling and solidity as seen in Fig. 4. Solidity cannot be changed without affecting the other dynamic-scaling parameters, and directly assessing the effect of each independently is difficult and was not accomplished in the study presented in Refs. 1, 19, and 24. However, helicopter blade element theory suggests that normalizing the coefficients with solidity as done in Fig. 4 is likely to minimize the effects of changes in solidity, ${ }^{25}$ and a theoretical alteration of select data concluded the same finding in Ref. 24. Ref. 25 contains derivations showing that the quantities $C_{L} / \sigma$ and $C_{Q} / \sigma$ should be identical for rotors with various values of solidity provided that the airfoil coefficients are identical and that other assumptions are met. Therefore the majority of the change observed in Fig. 4, between model and full-scale results at the same Reynolds number can likely be attributed to changes in dynamic scaling.

4) Experimental Example of Dynamic Scaling Importance for Rotorcraft Stability

In the study of rotorcraft stability, dynamic scaling is of significant importance. ${ }^{20}$ Matching reduced frequency (advance ratio), mass ratio (Lock number), and Froude number often takes precedence over matching Mach number even though blade tip Mach numbers can exceed 0.9 (Ref. 20). Ref. 22 presents the results of a dynamic stability test of the V22 tiltrotor in TDT that was Mach-scaled and dynamically-scaled $(M$, $\varepsilon, k, \mu_{m}$, and $F_{r}$ ) for R-134a. With air as a test medium, Mach number and mass ratio were no longer scaled. In this study, the prop-rotor pylon instability boundary was tested in both mediums. Fig. 5 contains a typical result showing a significantly different and un-conservative boundary obtained in air by comparison to the data acquired in R-134a. In Fig. 5, a dynamic instability is depicted as a negative value of damping with zero damping representing the boundary between stable and unstable.

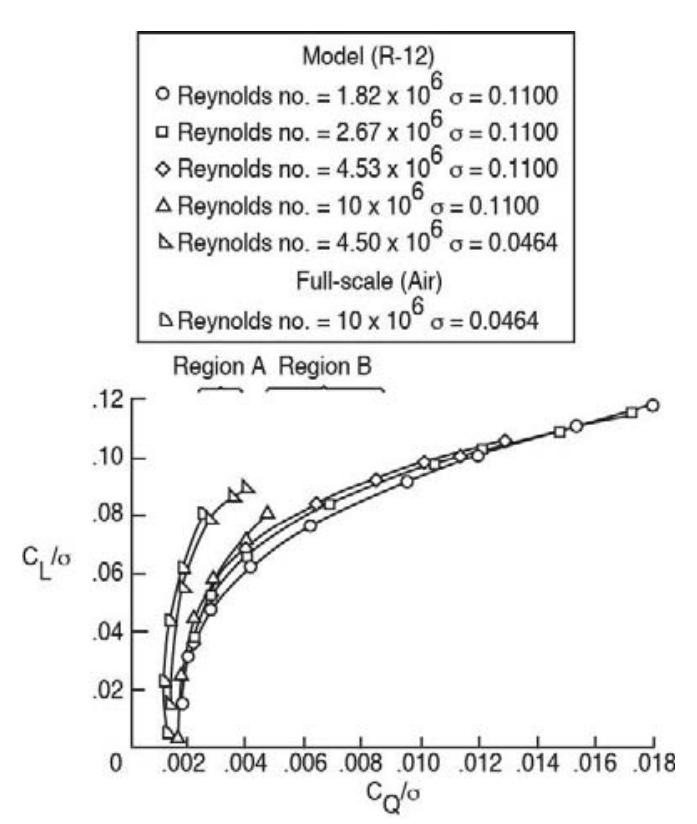

Figure 4. Rotorcraft performance comparison, sub-scale TDT data acquired in heavy gas and full-scale NFAC data acquired in air. Courtesy of Ref. 1

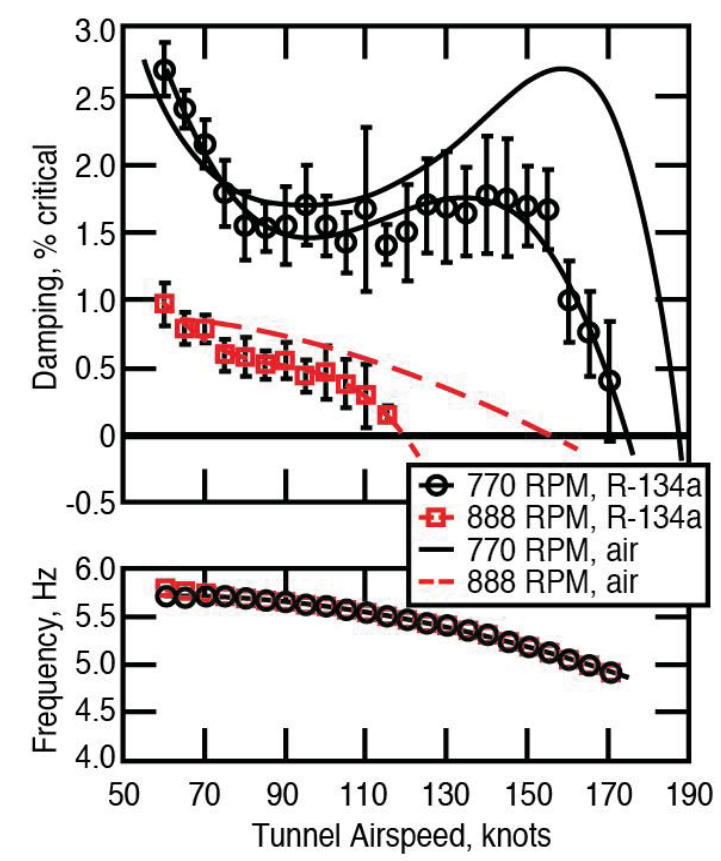

Figure 5. Tilt-rotor stability boundary variation, comparison of results obtained in R-134a and air. Courtesy of Ref. 4

$\S$ The TDT test described in Ref. 24 utilized R-12 heavy gas in lieu of the currently available R-134a. Originally designed for using R-12, TDT was converted to an R-134a facility for environmental reasons. R-12 offers very similar and slightly better scaling relationships than R-134a. 
5) Summary of Dynamic Scaling Importance

The analytical and experimental results presented here underscore the importance of proper dynamic scaling. Testing a model that is not properly scaled may produce meaningful results; however, those results could also be misleading when characterizing the behavior of a particular flight vehicle. Therefore, to investigate a dynamic aeroelastic event occurring on a flight vehicle such as flutter, gust load alleviation, ride quality, aeroservoelasticity, dynamic buffet, and launch vehicle ground wind loads; it is critical to use a dynamically-scaled model.

\section{Aeroelastic Scaling Capability of TDT}

\section{A. R-134a Gas Properties}

TDT can use air or tetrafluoroethane, R-134a, as its test medium enabling unique capabilities for providing dynamic similitude to full-scale vehicles. A colorless gas, R-134a has a density of approximately 3.5 times that of air, a viscosity of approximately $2 / 3$ that of air, and a speed of sound of approximately $1 / 2$ that of air for similar temperatures and pressures. Additionally, the ratio of specific heats $(\gamma)$ of R-134a is approximately 1.11 (Ref. 15).

\section{B. Dynamic Aeroelastic Scaling Capability}

To quantify the unique capabilities of the TDT, consider the scaling requirements of a compressible flow dynamically-scaled fixed-wing model described by Eqs. (1, 4, and 5). The model-scale to full-scale ratio of any parameter can be denoted by the symbol $\lambda_{\text {variable }}$ where variable is the structural or gas property being scaled. For this example, model velocity is determined by Mach number and speed of sound $(a)$. Therefore, the velocity scale is:

$$
\lambda_{\mathrm{V}}=\lambda_{a}
$$

Satisfying mass ratio (Eq. 5), the required flow density $(\rho)$ is determined by the cube of the length scale and the corresponding model weight as shown in Eq. (9).

$$
\lambda_{\rho}=\frac{\lambda_{\text {weight }}}{\lambda_{L}{ }^{3}}
$$

Satisfying reduced frequency (Eq. 4), the required structural frequencies of the model are determined by length and velocity scale as shown in Eq. (10).

$$
\lambda_{\omega}=\lambda_{a} / \lambda_{L}
$$

From the relationships shown in Eqs. (8-10), the selection of model weight or length scale appears to be arbitrary. However, the required structural weight will be specified by frequency requirements, chosen material, and model design parameters. From cantilever beam theory, ${ }^{26}$ the natural frequency is proportional to material and structural relationships as shown in Eq. (11), defined here as the structural constraint equation. The structural constraint equation will then dictate the structural mass and required model weight. With the introduction of the structural constraint equation, Eqs. (4, 5, and 11) can now be combined to derive Eq. (3) showing that a dynamically-scaled model is also elastically-scaled. When no other scaling requirement exists for model length, the length scale is typically chosen to be as large as possible to facilitate model construction, instrumentation installation, and to increase Reynolds number.

$$
\omega^{2} \propto \frac{E I}{m L^{4}}
$$

From inspection of the relations shown in Eq. (8) and Eq. (10), one can see that a slower speed of sound and larger model will yield a lower required model frequency and flow velocity. Lower required frequencies are advantageous for model design since reducing the effective stiffness of a structure is relatively easy whereas 
increasing the stiffness for the selected materials may not be possible. Additionally, the ability to create higher flow densities is advantageous since adding ballast weight to match full-scale mass similitude and center of gravity (C.G.) location is relatively easy, however, removing weight from a structure may not be possible. Finally, a lower required flow velocity equates to a lower dynamic pressure and corresponding loads. Material stress resulting from aerodynamic loads is directly proportional to dynamic pressure and for otherwise similar designs is independent of length scale; however, the length scale may affect the required dynamic pressure. This is demonstrated in Eq. (12) for an example of bending stress resulting from lift.

$$
\begin{aligned}
& \sigma_{\text {stress }}=\frac{M_{\text {bend }} y}{I}, \text { where } \\
& I \propto L^{4} \\
& y \propto L \\
& M_{\text {bend }} \propto q C_{L} S \cdot L \propto q C_{L} L^{2} \cdot L \\
& \therefore \\
& \sigma_{\text {stress }} \propto \frac{q C_{L} L^{3} \cdot L}{L^{4}} \\
& \sigma_{\text {stress }} \propto q C_{L}
\end{aligned}
$$

For dynamically-scaled tests, matching Reynolds number is typically not possible as flow density is driven by other scaling laws, and length scale is limited by the size of the wind tunnel. In order to analyze dynamic scaling limitations, further analysis of the structural constraint equation, Eq. (11), is required. For a fixed geometric design, $I$ is proportional to $L^{4}$ and $m$ is proportional to $\rho_{s} L^{2}$, where $\rho_{s}$ is the material density. In order to separate the dependence upon length from changes in geometric design, two new scaling parameters $\lambda_{G E O}$ and $\lambda_{B A L L A S T}$ are introduced that represent model design features. $\lambda_{G E O}$ is the model to full-scale ratio of the change in $I$, independent of length, due to geometric design of the beam structure such as altering the spar cap to spar web thickness ratio, or altering the beam selection from an I-beam to a box-beam and so forth. Similarly, $\lambda_{B A L L A S T}$ is the model to full-scale ratio of non-structural weight per unit length, also independent of the length scale. $\lambda_{B A L L A S T}$ is altered by design choices such as the installation of ballast weights or exclusion of fuel weight. $\lambda_{G E O}$ and $\lambda_{B A L L A S T}$ are defined as shown in Eq. (13) and Eq. (14) respectively.

$$
\begin{gathered}
\lambda_{I}=\lambda_{G E O} \lambda_{L}{ }^{4} \\
\lambda_{m}=\lambda_{B A L L A S T} \lambda_{\rho_{s}} \lambda_{L}{ }^{2}
\end{gathered}
$$

Full-scale aircraft employ complicated design features to maximize strength and stiffness for minimal mass such as lightening holes, tailored spar designs, and tailored skin thickness. Replicating these complex geometries in a simplified wind tunnel model is not practical and often times not possible due to the physical size limitations or the minimum gage restrictions of the skin. Similarly, adding non-structural weight to a model is easy whereas removing weight from a structure meeting a required $I$ is not possible. The only potential for a reduction of non-structural weight is the exclusion of fuel in a wind tunnel model. However, typical model design techniques require substantially more structural weight for a required $I$ than utilized for full-scale aircraft. Therefore, matching the structural efficiency with a wind tunnel model is difficult. With the introduction of these two parameters, the structural constraint equation, Eq. (11), can be combined with Mach number and reduced frequency, Eq. (1) and Eq. (4), and expanded as shown in Eq. (15). This expansion then separates the dependence of structural stiffness (EI) and mass distribution $(m)$ upon $L$ which would otherwise result in misleading scale relationships. 


$$
\begin{aligned}
& \lambda_{\omega}{ }^{2}=\frac{\lambda_{E} \lambda_{I}}{\lambda_{m} \lambda_{L}{ }^{4}} \\
& \lambda_{\omega}{ }^{2}=\frac{\lambda_{E} \lambda_{G E O} \lambda_{L}{ }^{4}}{\lambda_{B A L L A S T} \lambda_{\rho_{s}} \lambda_{L}{ }^{2} \lambda_{L}{ }^{4}} \\
& \frac{\lambda_{a}^{2}}{\lambda_{L}{ }^{2}}=\frac{\lambda_{E} \lambda_{G E O} \lambda_{L}{ }^{4}}{\lambda_{B A L L A S T} \lambda_{\rho_{s}} \lambda_{L}{ }^{2} \lambda_{L}{ }^{4}} \\
& \therefore \\
& \lambda_{a}{ }^{2}=\frac{\lambda_{E} \lambda_{G E O}}{\lambda_{\rho_{s}} \lambda_{B A L L A S T}}=\frac{\lambda_{G E O}}{\lambda_{B A L L A S T}} \lambda_{\frac{E}{\rho_{s}}}
\end{aligned}
$$

Analysis of Eq. (15) reveals that for a fixed model design, the scale of speed of sound dictates the scale of the ratio of material stiffness to material density. These required structural properties are independent of length scale or tunnel pressure.

In order to satisfy mass ratio, the required wind tunnel density scale can then be determined by combining Eqs. $(5,14)$ with Eq. (15) as done by Eq. (16).

$$
\lambda_{\rho}=\lambda_{\rho_{s}} \lambda_{B A L L A S T}=\frac{\lambda_{E} \lambda_{G E O}}{\lambda_{a}^{2}}
$$

The consequence of dynamic scaling upon Reynolds number scale is shown in Eq. (17): that Reynolds number is not a function of wind tunnel capability, but rather the gas properties, material selection, and the structural constraint equation. As a result, a dynamically-scaled model utilizing the same geometric design will have a higher Reynolds number when tested in TDT than any cryogenic wind tunnel or any air wind tunnel of comparable size. For purposes of dynamic scaling, recall that flow density is not an independent quantity (Eq. 16). This consequence of dynamic scaling should not be confused with the performance testing capability of a model when dynamic scaling can be relaxed. For example, performance-based testing can better address Reynolds number effects with specialized facilities such as NTF or ETW.

$$
\lambda_{R e}=\frac{\lambda_{\rho} \lambda_{V} \lambda_{L}}{\lambda_{\mu}}=\frac{\lambda_{E} \lambda_{G E O}}{\lambda_{a}{ }^{2}} \frac{\lambda_{a} \lambda_{L}}{\lambda_{\mu}}=\frac{\lambda_{E} \lambda_{G E O} \lambda_{L}}{\lambda_{a} \lambda_{\mu}}
$$

Table 3 shows a summary of the scale factors for a dynamically-scaled model that is also Mach-scaled to a vehicle at 30,000 ft for TDT in R-134a, S1MA in air, and NTF in cryogenic $\mathrm{N}_{2}$. S1MA was chosen for comparison in Table 3 since it is the largest transonic facility, and similarly, NTF is the largest and coldest cryogenic facility. Since density and the corresponding pressure are not independent quantities, it is assumed that the wind tunnel density could be adjusted to match the required value for purposes of this gas/structural-property comparison. Therefore, the dynamic-scaling capability of all the other wind tunnels introduced in Table 1 will be less capable than those shown in Table 3 regardless of pressure capability. 
Table 3. Scale Factors; Dynamically-Scaled, Mach-Scaled Model (simulated to 30,000 ft)

\begin{tabular}{|c|c|c|c|c|c|}
\hline Scale Factor & $\begin{array}{l}\text { Governing } \\
\text { Factors }\end{array}$ & Scale Relation & $\begin{array}{c}\text { TDT } \\
\text { (R-134a) }\end{array}$ & $\begin{array}{l}\text { S1MA } \\
\text { (Air) }\end{array}$ & $\begin{array}{c}\text { NTF } \\
\left(\text { Cryo N }_{2}\right)\end{array}$ \\
\hline Length-scale & $\begin{array}{l}\text { geometric } \\
\text { constraints }\end{array}$ & $\begin{array}{c}\text { use TDT as ref. } \\
\text { length }\end{array}$ & 1 & 1.406 & 0.513 \\
\hline Velocity-scale & $\begin{array}{l}M \text {, speed of } \\
\text { sound }\end{array}$ & $\lambda_{V}=\lambda_{a}$ & 0.544 & $1.166 *$ & 0.712 \\
\hline Frequency-scale & $\begin{array}{c}k \text {, speed of } \\
\text { sound, length- } \\
\text { scale }\end{array}$ & $\lambda_{\omega}=\lambda_{a} / \lambda_{L}$ & 0.544 & 0.829 & 1.388 \\
\hline Viscosity-scale & fluid properties & $\lambda_{\mu}$ & 0.836 & 1.273 & 0.543 \\
\hline $\begin{array}{l}\text { Structural Stiffness / } \\
\text { Structural Density - } \\
\text { scale }\end{array}$ & $\begin{array}{l}\text { struct. constr. } \\
\text { eqn., } M, k\end{array}$ & $\begin{array}{c}\lambda_{E / \rho_{s}}= \\
\lambda_{a}^{2} \lambda_{B A L L A S T} / \lambda_{G E O}\end{array}$ & $\begin{array}{c}0.296 \lambda_{B A L L A S T} \\
/ \lambda_{G E O}\end{array}$ & $\begin{array}{c}1.360 \lambda_{B A L L A S T} \\
/ \lambda_{G E O}\end{array}$ & $\begin{array}{c}0.507 \lambda_{B A L L A S T} \\
/ \lambda_{G E O}\end{array}$ \\
\hline Density-scale & $\begin{array}{l}\text { struct. constr. } \\
\text { eqn., } M, k, \mu_{m}\end{array}$ & $\lambda_{\rho}=\lambda_{E} \lambda_{G E O} / \lambda_{a}^{2}$ & $\lambda_{E} \lambda_{G E O} / 0.296$ & $\lambda_{E} \lambda_{G E O} / 1.360$ & $\lambda_{E} \lambda_{G E O} / 0.507$ \\
\hline Reynolds-scale & $\begin{array}{l}\text { struct. constr. } \\
\text { eqn., } M, k, \mu_{m}, \\
\text { Fluid prop. }\end{array}$ & Eq. (17) & $2.199 \lambda_{E} \lambda_{G E O}$ & $0.947 \lambda_{E} \lambda_{G E O}$ & $1.327 \lambda_{E} \lambda_{G E O}$ \\
\hline
\end{tabular}

*Air wind tunnels typically run at temperatures significantly warmer than the standard atmospheric temperature of $411.86^{\circ} \mathrm{R}$ or $-47.81^{\circ} \mathrm{F}$ at $30,000 \mathrm{ft}$; a tunnel temperature of $100^{\circ} \mathrm{F}$ was assumed for TDT and S1MA, and $-250^{\circ} \mathrm{F}$ for NTF

A significant characteristic of the scale factors presented in Table 3 is the relation of the structural stiffness to structural density $\left(\lambda_{E / \rho_{s}}\right)$, also known as specific modulus. The required specific modulus is a function of the $\lambda_{B A L L A S T} / \lambda_{\text {GEO }}$ ratio. An example scaling exercise is included in Appendix A showing the difficulty in achieving a $\lambda_{B A L L A S T} / \lambda_{G E O}$ ratio of less than three for a scaled transport wing using typical model construction techniques. Therefore, in order to match Mach number and reduced frequency, any model dynamically-scaled for air requires the use of material with a significantly higher specific modulus than the aircraft structure to be simulated, or atypical construction techniques. This relation holds regardless of the length scale of the model. Similarly, it is likely that materials with a higher specific modulus will also be required for dynamically-scaled testing in cryogenic $\mathrm{N}_{2}$.

A summary of the stiffness and density values for typical materials are shown in Table 4, and they are available from various material references. Heat treating and other manufacturing treatments have substantial effect upon the yield or ultimate strength of metals; however, these treatments have little effect upon the stiffness or density of the material. Most importantly, the specific modulus is nearly the same for nearly all metals to include aircraft aluminum, stainless steel, magnesium, and specialized steels such as Vascomax. The only material that offers an increase in the specific modulus over aircraft aluminum is carbon fiber. Therefore, dynamic simulation of an aluminum wing in an air wind tunnel requires the use of carbon fiber as the primary model structure. This complicates the design and construction of stiffness-and-mass-tailored models. Also, the ability to realize the stiffness to density gain may be dependent upon the minimum gage of the material. Sub-scale dynamically-scaled tests are also possible in cryogenic $\mathrm{N}_{2}$ based solely upon the material properties and scaling ratios presented in this paper. However, cryogenic testing presents additional challenges such as thermal expansion/contraction of model materials, and variable material elasticity as a function of temperature. ${ }^{27}$ This would likely require a dynamically-scaled model to be constructed solely from carbon-fiber with tailored spar and skin thicknesses. Additionally, model verification with ground vibration testing is increasingly difficult since the roomtemperature response of the model will likely be different than the response at cryogenic temperatures. Design and construction of such a model would be complex and costly by

Table 4. Typical Material Properties

\begin{tabular}{|l|c|c|c|}
\hline Material & $\begin{array}{c}\text { Young's } \\
\text { Modulus } \\
\begin{array}{l}\boldsymbol{E} \\
\left(\mathbf{l b s} / \mathbf{i n}^{2}\right)\end{array}\end{array}$ & $\begin{array}{c}\text { Density } \\
\boldsymbol{\rho}_{\boldsymbol{s}} \\
\left.\mathbf{l l b s}^{\mathbf{l}} \mathbf{i n}^{3}\right)\end{array}$ & $\begin{array}{c}\text { Specific } \\
\text { Modulus } \\
\boldsymbol{E} / \boldsymbol{\rho}_{\boldsymbol{s}} \text { (in) }\end{array}$ \\
\hline $\begin{array}{l}\text { Aluminum } \\
(2024)\end{array}$ & $1.06 \times 10^{7}$ & 0.101 & $1.06 \times 10^{8}$ \\
\hline Steel A36 & $2.90 \times 10^{7}$ & 0.280 & $1.04 \times 10^{8}$ \\
\hline $\begin{array}{l}\text { Stainless } \\
\text { Steel T400 }\end{array}$ & $2.89 \times 10^{7}$ & 0.280 & $1.03 \times 10^{8}$ \\
\hline Vascomax & $2.90 \times 10^{7}$ & 0.290 & $0.99 \times 10^{8}$ \\
\hline $\begin{array}{l}\text { Magnesium } \\
\text { Am1004 }\end{array}$ & $0.65 \times 10^{7}$ & 0.066 & $0.98 \times 10^{8}$ \\
\hline Titanium & $1.52 \times 10^{7}$ & 0.163 & $0.93 \times 10^{8}$ \\
\hline Fiberglass* & $0.37 \times 10^{7}$ & 0.067 & $0.55 \times 10^{8}$ \\
\hline $\begin{array}{l}\text { Carbon } \\
\text { Fiber* }\end{array}$ & $3.35 \times 10^{7}$ & 0.064 & $5.21 \times 10^{8}$ \\
\hline
\end{tabular}

*material properties of composite materials are heavily dependent upon the construction technique, resins used, and ply orientation; values presented here are representative only 
comparison to an aluminum/foam model constructed for TDT. If the flight vehicle to be simulated is constructed from carbon fiber, then it is likely that sub-scale simulation is only possible in TDT.

Another important characteristic of the scale factors shown in Table 3 is the relation of required frequency for the various test mediums. An often overlooked benefit of R-134a is that lower frequency modes offer substantially greater model safety during dynamic testing. The time required to reach large amplitudes of any unstable event is increased as frequency is reduced thereby enabling longer reaction time by the test team. This feature of R-134a couples well with the other built-in safety features of TDT such as the bypass valves, observation windows, and model catch screen in order to significantly reduce the risk of dynamic testing.

\section{Froude-Scale and Mach-Scale Capability}

Froude-scaled models, related by Eq. (6) and assuming that gravity is constant, require that length scale is a function of velocity as expressed in Eq. (18). If Mach number must also be matched, such as a rotorcraft test, then $\lambda_{V}$ can be replaced by $\lambda_{a}$ in Eq. (18). Therefore, the required length-scale is equal to the square of the scale of speed of sound. Any air wind tunnel will require a length scale equal to or larger than the full-scale vehicle in order to satisfy this requirement regardless of the density or pressure capability. R-134a, however, offers the possibility to test subscale models since the speed of sound is approximately half that of air which yields a required length scale of approximately 0.25 depending upon the details of the desired atmospheric conditions to be simulated. Cryogenic $\mathrm{N}_{2}$ tunnels also offer some benefit to Froude number scaling since the cold temperatures slow the speed of sound of $\mathrm{N}_{2}$, but not to the extent experienced with R-134a. For example, at $-250^{\circ} \mathrm{F}$, the speed of sound scale is approximately 0.63 for standard sea level air or approximately 0.71 for air at 30,000 ft. This yields a required length scale of approximately 0.4 to 0.5 .

$$
\lambda_{L}=\lambda_{V}^{2}
$$

A summary of the scale factors for a Froude-scaled, Mach-scaled model is presented in Table 5 for a vehicle flying at sea level. The scaling parameters achievable in TDT are shown along with the comparable scaling parameters required for testing in the S1MA with air, and NTF with cryogenic $\mathrm{N}_{2}$ at $-250^{\circ} \mathrm{F}$.

Table 5. Dynamic Aeroelastic Scaling Factors, Froude-Scaled and Mach-Scaled Model (simulated to sea level).

\begin{tabular}{|c|c|c|c|c|c|}
\hline Scaling Parameter & $\begin{array}{c}\text { Governing } \\
\text { Factors }\end{array}$ & Scale Relation & $\begin{array}{c}\text { TDT } \\
\text { (R-134a) }\end{array}$ & $\begin{array}{c}\text { S1MA } \\
\text { (Air) }\end{array}$ & $\begin{array}{c}\text { NTF } \\
\left(\mathbf{C r y o ~ N}_{\mathbf{2}} \text { ) }\right.\end{array}$ \\
\hline Velocity-scale & $\begin{array}{c}\text { Mach, speed of } \\
\text { sound }\end{array}$ & $\lambda_{V}=\lambda_{a}$ & 0.485 & 1.039 & 0.635 \\
\hline Length-scale & Froude number & $\lambda_{L}=\lambda_{a}{ }^{2}$ & 0.235 & 1.080 & 0.403 \\
\hline $\begin{array}{c}\text { Max rotor-span } \\
\text { (model-scale) }\end{array}$ & Test section size & $\begin{array}{c}63 \% \text { of test } \\
\text { section* }\end{array}$ & $10 \mathrm{ft}$ & $14.17 \mathrm{ft}$ & $5.17 \mathrm{ft}$ \\
\hline \hline $\begin{array}{c}\text { Max simulated } \\
\text { rotor-span } \\
\text { (full-scale) }\end{array}$ & $\begin{array}{c}\text { Froude number, } \\
\text { vehicle size }\end{array}$ & & $42.6 \mathrm{ft}$ & $13.12 \mathrm{ft}$ & $12.8 \mathrm{ft}$ \\
\hline
\end{tabular}

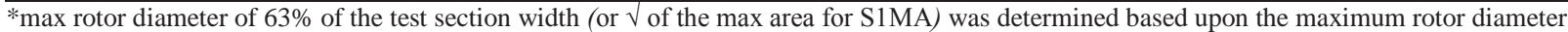
historically used in TDT

Fig. 6 shows the Froude-scaled Mach-scaled boundary for rotorcraft, where Mach-scaling is important even for near-zero velocity as this defines the tip Mach number of the rotor disk. Although included in Fig. 6 for comparison, it is unlikely that rotorcraft mechanical and instrumentation components can survive the cryogenic temperatures of NTF while running at high rpm. Rotor diameter and Mach envelopes of common rotorcraft are also included in Fig. 6. Large rotorcraft exceed the scaling capability of TDT, however, TDT offers the closest Froude number similitude. 


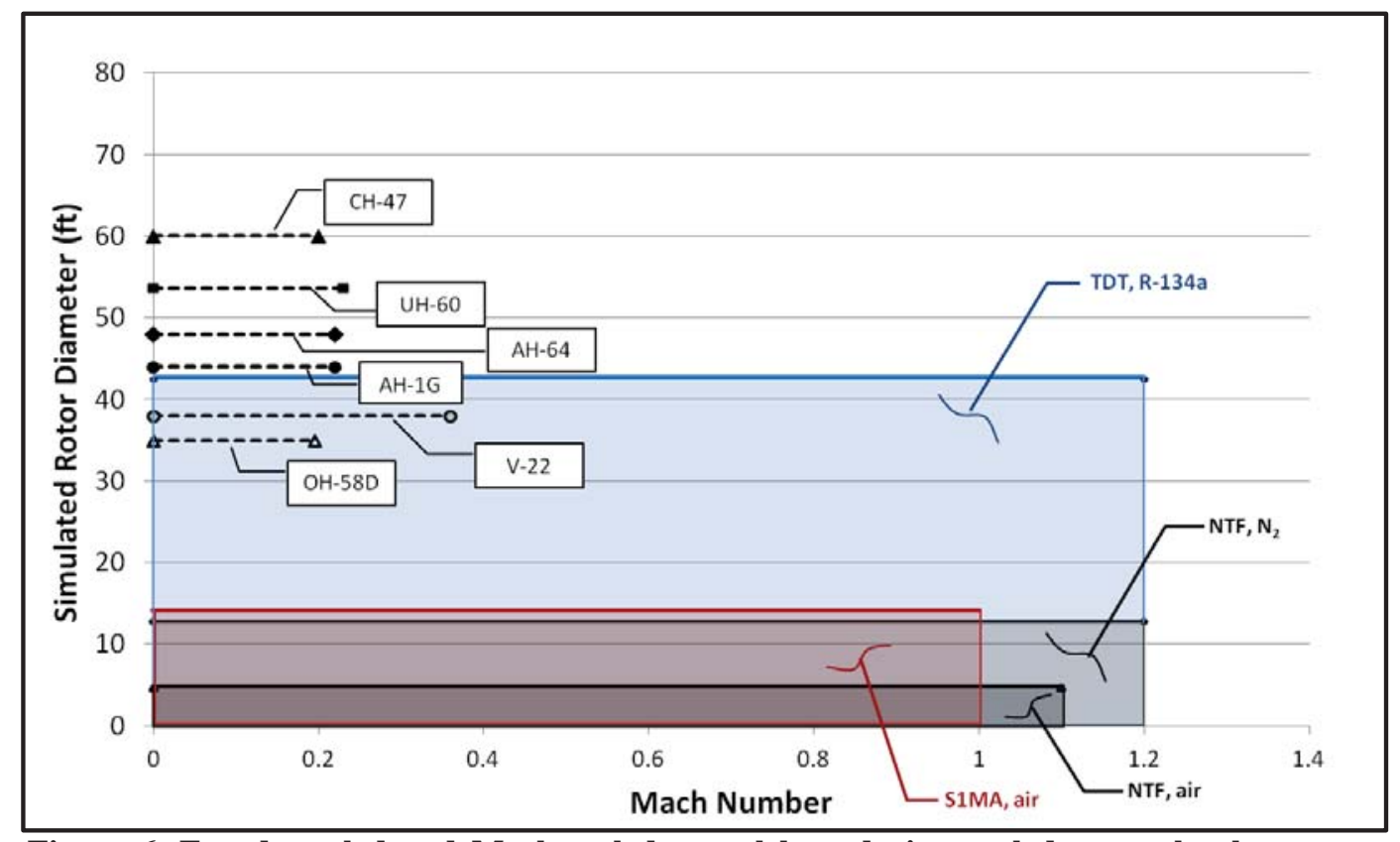

Figure 6. Froude-scaled and Mach-scaled tunnel boundaries, scaled to sea level, and example rotorcraft operating boundaries

\section{TDT Flow Quality and Reynolds Number Capability}

An often overlooked requirement for conducting dynamic aeroelastic testing is that wind tunnel turbulence and model support system-related buffeting needs to be at acceptably low levels. Dynamically-scaled models are often extremely flexible and fragile by comparison to typical aerodynamic performance models. Therefore, a benefit of the features of TDT is that the flow quality lends itself to the potential extension of an increased role in aerodynamic performance testing. It is beyond the scope of this paper to validate the capability of TDT to conduct aerodynamic performance tests, but the results of an initial assessment look promising. A survey of many transonic wind tunnels in the United States and Europe was conducted in the 1970s showing that TDT had one of the lowest turbulence levels by comparison to other transonic facilities surveyed at the time. ${ }^{28,29}$ A review of these results in addition to more recent studies is underway to fully evaluate the flow quality of TDT by comparison to other transonic wind tunnels of similar size. This ongoing study is intended to include an analysis of tunnel parameter uncertainty, ${ }^{30}$ data repeatability, turbulence, ${ }^{14,28,29,31-35}$ flow angularity, ${ }^{36-38}$ wall interference, ${ }^{37-45}$ boundary layer, ${ }^{14,46}$ Mach number distribution, ${ }^{14,30,47}$ and transonic similarity. ${ }^{15,16,48}$

It should also be noted that the Reynolds number capability of TDT is higher than most transonic wind tunnels surpassed only by the large cryogenic facilities of NTF and ETW as shown in Table 1. It is also notable that this Reynolds number is achieved with a lower dynamic pressure than achieved in air or cryogenic tunnels resulting in lower model loads and corresponding elastic deflection. Lower dynamic pressure also requires less tunnel drive power, and less model power for rotorcraft tests.

\section{Role of TDT in Supporting Future NASA Missions}

With advancements in CFD capabilities, the role of wind tunnel testing has been altered in recent decades to achieve a new balance among computation, experiment, and analysis. ${ }^{48}$ Modern flight vehicles are increasingly more complex with a focus on weight reduction in addition to an increased use of active controls and structural tailoring. These design thrusts typically result in increasingly flexible aircraft with a higher propensity for fluid-structure interactions. Additionally, tilt-rotor designs have not yet matured and offer the potential for improvement to the national airspace system in addition to tactical military advantages. ${ }^{49}$

In addition to understanding a particular complex problem of a new vehicle, there are several other types of investigations that also warrant the need for experimental research. Such investigations include but are not limited to: 
1. Those investigations that exceed the capability of CFD such as ground wind loads investigations for launch vehicles.

2. Radically new designs of vehicles that are outside of the traditional design space such as morphing aircraft concepts.

3. Tests of multiple variations in configurations or conditions where wind tunnel testing is more efficient and timely than CFD for building a database.

4. Demonstration of a new technology, such as active controls. Wind tunnel testing of these concepts increases the technology readiness level at a lower risk than a flight vehicle program and offers the ability to isolate and control individual variables.

The ability to understand and solve complex problems associated with advanced aircraft is dependent upon the utilization of complex and capable experimental facilities. TDT has been used extensively in the investigation of ground wind loads due to its ability to match reduced frequency and at times the full-scale Reynolds number of launch vehicles on the pad. ${ }^{3,51,52}$ It has also been used to investigate radical designs such as morphing aircraft due to its high-risk capability, Mach number range, and size. ${ }^{53,54}$ Finally, active control tests have also been conducted to investigate gust load alleviation and aeroelastic stability augmentation due to TDT's high risk capabilities and dynamic scaling abilities. ${ }^{55-58}$

\section{Concluding Remarks}

In this paper, an overview of typical scaling parameters that define aeroelastic similitude has been presented. Theoretical exercises and experimental results have also been presented emphasizing the importance of certain scaling parameters during aeroelastic investigations. A systematic analysis was done highlighting the abilities of TDT to match these scaling parameters for many classes of flight vehicles. For comparison, many of the world's leading transonic facilities where briefly discussed, and the scaling parameters where also analyzed for application within these facilities. From this analysis, it is shown that TDT is uniquely capable of conducting dynamicallyscaled aeroelastic tests with a sub-scale model using conventional construction techniques and materials. The TDT is also well-suited for conducting static aeroelastic tests, high-risk configuration testing, and many other classes of investigations that study unsteady flow phenomena or require unusual model support systems. To complement the dynamic and often high-risk aeroelastic testing, the TDT offers many features that reduce risk and complement dynamic investigations.

In addition to its aeroelastic capabilities, a preliminary evaluation of TDT's flow quality was conducted. The initial results look promising and warrant further investigation to consider the use of TDT for aerodynamic performance testing.

Finally, given the anticipated future aeronautic research thrusts of NASA, future air vehicles are likely to be increasingly flexible with potentially radical design. New technologies are also likely to require the design, improvement, and/or validation of active control technologies. These anticipated designs highlight the need for a test facility capable of conducting aeroelastically-scaled or high-risk tests. With the unique and diverse capabilities of the TDT, it is expected that TDT will continue to play a vital role in the research and development of innovative vehicles for many years as it has done for the past five decades.

\section{References}

${ }^{1}$ Yeager, W. T; Kvaternik, R. G.; “A Historical Overview of Aeroelasticity Branch and Transonic Dynamics Tunnel Contributions to Rotorcraft Technology and Development;” NASA TM-2001-211054; August 2001.

${ }^{2}$ Cole, S. R.; Noll, T. E.; Perry III, B.; "Transonic Dynamics Tunnel Aeroelastic Testing in Support of Aircraft Development;" Journal of Aircraft, Vol. 40, No. 5, September-October 2003.

${ }^{3}$ Cole, S. R.; Keller, D. K.; Piatak, D. J.; "Contributions of the NASA Langley Transonic Dynamics Tunnel to Launch Vehicle and Spacecraft Development;” AIAA Dynamics Specialists Conference, Atlanta, GA, April 2000, AIAA $2000-1772$.

${ }^{4}$ Perry III, B.; Noll, T. E.; Scott, R. C.; "Contributions of the Transonic Dynamics Tunnel to the Testing of Active Control of Aeroelastic Response;” AIAA Dynamic Specialists Conference, Atlanta, GA, April 2000, AIAA 2000-1769.

${ }^{5}$ Fung, Y. C.; An Introduction to the Theory of Aeroelasticity; Dover Publications, New York, NY; 1993.

${ }^{6}$ Bisplinghoff, R. L.; Ashley, H.; and Halfman, R. L.; Aeroelasticity; Addison-Wesley Publishing Company, Inc., Cambridge MA; 1955; renewed 1983 by Bisplinghoff, Ashley, and Halfman.

${ }^{7}$ Cole, H. A.; Erickson, A. L.; and Rainey, A. G.; "Buffeting During Atmospheric Ascent;" NASA Space Vehicle Design Criteria (Structures), NASA SP 8001; May 1964, Revised November 1970. 
${ }^{8}$ Garretson, D.; Mair, H.; Martin, C.; Sullivan, K.; Teichman, J.; "Review of CFD Capabilities;" Institute for Defense Analysis, Science and Technology Policy Institute; Washington, D.C.; November 2005.

${ }^{9}$ Anton, P. S.; Gritton, E. C.; Mesic, R.; Steinberg, P.; Johnson, D.; Block, M.; Brown, M.; Drezner, J.; Dryden, J.; Hamilton, T.; Hogan, T.; Peetz, D.; Raman, R.; Strong, J.; Trimble, W.; "Wind Tunnel and Propulsion Test Facilities, an Assessment of NASA's Capabilities to Serve National Needs;" The RAND Corporation, Santa Monica, CA; 2004.

${ }^{10}$ Anton, P. S.; Johnson, D. J.; Block, M.; Brown, M.; Drezner, J.; Dryden, J.; Gritton, E. C.; Hamilton, T.; Hogan, T.; Mesic, R.; Peetz, D.; Raman, R.; Steinberg, P.; Strong, J.; Trimble, W.; "Wind Tunnel and Propulsion Test Facilities, Supporting Analyses to an Assessment of NASA's Capabilities to Serve National Needs;" The RAND Corporation, Santa Monica, CA; 2004.

${ }^{11}$ Anton, P. S.; Raman, R.; Osburg, J.; Kallimani, J. G.; “An Update of the Nation’s Long-Term Strategic Needs for NASA's Aeronautics Test Facilities;" The RAND Corporation, Santa Monica, CA; 2009.

${ }^{12}$ Under Secretary of Defense (Acquisition, Technology and Logistics); "NASA Aeronautics Facilities Critical to DoD Report to Congress;" Department of Defense, 2007.

${ }^{13}$ Cole, S. R.; Garcia, J. L.; "Past, Present and Future Capabilities of the Langley Transonic Dynamics Tunnel from an Aeroelasticity Perspective;" AIAA Dynamics Specialists Conference, Atlanta, GA; April 2000, AIAA 2000-1767.

${ }^{14}$ Staff of the Aeroelasticity Branch; "The Langley Transonic Dynamics Tunnel;" Langley Working Paper LWP-799, September 1969, reprinted July 1992.

${ }^{15}$ Kvaternik, R. G.; "Computer Programs for Calculating the Isentropic Flow Properties for Mixtures of R-134a and Air;" NASA Langley Research Center, Hampton, VA, NASA TM-2000-210622, November 2000.

${ }^{16}$ Anders, J. B.; Anderson, W. K.; Murthy, A.V.; "Transonic Similarity Theory Applied to a Supercritical Airfoil in Heavy Gas;" Journal of Aircraft, Vol. 36, No. 6, November - December 1999.

${ }^{17}$ Pereira, P.; Almeida, L.; Suleman, A.; Bond, V.; Canfield, R.; and Blair, M.; "Aeroelastic Scaling and Optimization of a Joined-Wing Aircraft Concept;" AIAA MultiDisciplinary Design Optimization Specialists Conference, Honolulu, HI; April 2007, AIAA 2007-1889.

${ }^{18}$ Heeg, J.; Spain, C. V.; Rivera, J. A.; "Wind Tunnel to Atmospheric Mapping for Static Aeroelastic Scaling;" $45^{\text {th }}$ AIAA Structural Dynamics and Materials Conference, Palm Springs, CA, April 2004, AIAA 2004-2044.

${ }^{19}$ Yeager, W. T.; Mirick, P. H.; Hamouda, M. H.; Wilbur, M. L.; Singleton, J. D.; Wilkie, W. K.; "Recent Rotorcraft Aeroelastic Testing in the Langley Transonic Dynamics Tunnel;" Alternate paper at the $47^{\text {th }}$ Annual Forum of the American Helicopter Society, Phoenix, AZ, May 1991.

${ }^{20}$ Hunt, G. K.; "Similarity Requirements for Aeroelastic Models of Helicopter Rotors;" Royal Aircraft Establishment (RAE); Farnborough, C.P. No. 1245, January 1972.

${ }^{21}$ Young, C. P.; Hergert, D. W.; Butler, T. W.; Herring, F. M.; "Buffet Test in the National Transonic Facility;" $17^{\text {th }}$ AIAA Aerospace Ground Testing Conference, Nashville, TN, July 1992, AIAA 92-4032.

${ }^{22}$ Piatak, D. J.; Kvaternik, R. G.; Nixon, M. W.; Langston, C. W.; Singleton, J. D.; Bennett, R. L.; Brown, R. K.; “A WindTunnel Parametric Investigation of Tiltrotor Whirl-Flutter Stability Boundaries;" AHS Annual Forum, Washington, DC, May 2001.

${ }^{23}$ Theodorsen, T.; Garrick, I. E.; "Mechanism of Flutter, a Theoretical and Experimental Investigation of the Flutter Problem;" NACA TR-685, 1940.

${ }^{24}$ Yeager, W. T.; Mantay, W. R.; "Correlation of Full-Scale Helicopter Rotor Performance in Air with Model-Scale Freon Data;" NASA TN D-8323, November 1976.

${ }^{25}$ Johnson, W.; Helicopter Theory; Dover Publications, Inc.; New York, NY; 1980.

${ }^{26}$ Meriovitch, L.; Elements of Vibration Analysis, second edition; McGraw-Hill; 1986.

${ }^{27}$ Cole, S. R.; "Exploratory Flutter Test in a Cryogenic Wind Tunnel;" Journal of Aircraft, Vol. 23, No. 12, December 1986.

${ }^{28}$ Whitfield, J. D.; Dougherty Jr., N. S.; "A Survey of Transition Research at AEDC;" Arnold Air Force Station, TN, July 1977.

${ }^{29}$ Dougherty Jr. N. S.; Steinle Jr., F. W.; "Transition Reynolds Number Comparisons in Several Major Transonic Tunnels;" AIAA paper No. 74-627, July 1974.

${ }^{30}$ Piatak, D. J.; "Survey of Primary Flow Measurement Parameters at the NASA Langley Transonic Dynamics Tunnel;" NASA TM-2003-212413, June 2003.

${ }^{31}$ Wieseman, C. D.; Sleeper, R. K.; "Measurements of Flow Turbulence in the NASA Langley Transonic Dynamics Tunnel;" NASA TM-2005-213529.

${ }^{32}$ Sleeper, R. K.; Keller, D. F.; Perry III, B.; Sandford, M. C.; "Characteristics of Vertical and Lateral Tunnel Turbulence Measured in Air in the Langley Transonic Dynamics Tunnel;" NASA TM-107734, March 1993.

${ }^{33}$ Amaya, M. A.; "Flow Quality Measurements in the NASA Ames Upgraded 11-by 11-ft Transonic Wind Tunnel;" 21st AIAA Aerodynamic Measurement Technology and Ground Testing Conference, Denver, CO, AIAA 2000-2681, June 2000.

${ }^{34}$ Igoe, W. B.; "Analysis of Fluctuating Static Pressure Measurements in the National Transonic Facility;" NASA TP-3475, March 1996

${ }^{35}$ King, R. A.; Andino, M. Y.; Melton, L.; Eppink, J.; Kegerise, M. A.; Tsoi, A.; "Flow Disturbance Characterization Measurements in the National Transonic Facility;" 50 th AIAA Aerospace Sciences Meeting, Nashville, TN, AIAA 2012-0104, January 2012.

${ }^{36}$ Yeager, W. T.; Wilbur, M. L.; Mirick, P. H.; Rivera Jr., J. A.; "Flow Angularity Measurements in the NASA-Langley Transonic Dynamics Tunnel;" NASA TM-2005-213946. 
${ }^{37}$ Krynytzky, A. J.; "Steady-State Wall Interference of a Symmetric Half-Model in the Langley Transonic Dynamics Tunnel;" 39th AIAA Aerospace Sciences Meeting and Exhibit, Reno, NV, January 2001, AIAA 2001-161.

${ }^{38}$ Krynytzky, A. J.; Johnsen, K. M.; Sommerfield, D. M.; "Uncertainty Evaluation of Wall Interference in a Large Transonic Wind Tunnel;" 27 $7^{\text {th }}$ AIAA Aerodynamic Measurement Technology and Ground Testing Conference, Chicago, IL, July 2010, AIAA 2010-4341.

${ }^{39}$ Rausch, R. D.; "Preliminary Wall Interference Assessment for NASA Langley Transonic Dynamics Tunnel;" Boeing Report DTF 26-3-07, October 1998.

${ }^{40}$ Baker, M.; Boyd, W.; Scott, R.; "High Speed Research;” Boeing Commercial Airplane Group, Boeing Phantom Works, Multi-Year Summary Report, Contract NAS1-20220, September 1999.

${ }^{41}$ Krynytzky, A. J.; "BYB-T-1710 Half-Model in the Langley Transonic Dynamics Tunnel (TDT), Test LD0533 Analysis Report;” Boeing Report B-2T66-M00-001, May 2000.

${ }^{42}$ Ewald, B. F. R., (Editor); "Wind Tunnel Wall Correction;" Advisory Group for Aerospace Research and Development (AGARD), AGARDograph 336, October 1998, CH3 and CH5.

${ }^{43}$ Mokry, M.; "Subsonic Wall Interference Corrections for Finite-Length Test Sections Using Boundary Pressure Measurements;" National Research Council, Ottawa, Ontario; K1A 0R6; AGARD CP-335, 1982.

${ }^{44}$ Cruz, J. R.; Mineck, R. E.; Keller, D. F.; Bobskill, M. V.; "Wind Tunnel Testing of Various Disk-Gap-Band Parachutes;" $17^{\text {th }}$ AIAA Aerodynamic Decelerator Systems Technology Conference and Seminar, May 2003, AIAA 2003-2129.

${ }^{45}$ Usry, J. W.; Wallace, J. W.; "Drag of a Supercritical Body of Revolution in Free Flight at Transonic Speeds and Comparison with Wind-Tunnel Data;" NASA TN-D-6580, December 1971.

${ }^{46}$ Wieseman, C. D.; Bennett, R. M.; "Wall Boundary Layer Measurements for the NASA Langley Transonic Dynamics Tunnel;" NASA TM-2007-214867, April 2007.

${ }^{47}$ Florance, J. R.; Rivera Jr., J. A.; "Sidewall Mach Number Distributions for the NASA Langley Transonic Dynamics Tunnel;" NASA TM-2001-211019.

${ }^{48}$ Wieseman, C. D.; Silva, W. A.; Spain, C. V.; Heeg, J.; "Transonic-Small-Disturbance and Linear Analyses for the Active Aeroelastic Wing Program;" $46^{\text {th }}$ AIAA Structures, Structural Dynamics, and Materials Conference, Austin, TX, April 2005, AIAA 2005-1995.

${ }^{49}$ Melanson, M. R.; Chang, M.; Baker, W. M.; "Wind Tunnel Testing's Future: A Vision of the Next Generation of Wind Tunnel Test Requirements and Facilities;" Lockheed Martin Aeronautics, Fort Worth TX, AIAA 2010-142.

${ }^{50}$ Stouffer, V.; Johnson, J.; and Gribko, J.; "Civil Tiltrotor Feasibility Study for the New York and Washington Terminal Areas;” Logisitics Management Institute, McLean, VA; NASA CR-2001-210659, January 2001.

${ }^{51}$ Keller, D. F.; Ivanco, T. G.; "Wind Tunnel Investigation of Ground Wind Loads for Ares Launch Vehicle;" NASA Langley Research Center; $28^{\text {th }}$ AIAA Applied Aerodynamics Conference, Chicago, IL June-July 2010 AIAA 2010-4371.

${ }^{52}$ Ivanco, T. G.; Keller, D. G.; "Investigation of Ground Wind Loads for Ares Launch Vehicles;” NASA Langley Research Center; Journal of Spacecraft and Rockets, Vol. 49, No. 4, July-August 2012.

${ }^{53}$ Ivanco, T. G.; Scott, R. C.; Love, M. H.; Zink, S.; Weisshaar, T. A.; "Validation of Lockheed Martin Morphing Concept with Wind Tunnel Testing;" 48 $8^{\text {th }}$ AIAA Structures, Structural Dynamics, and Materials Conference, Honolulu, HI, April 2007, AIAA 2007-2235.

${ }^{54}$ Love, M. H.; Zink, P. S.; Stroud, R. L.; Bye, D. R.; Rizk, S.; White, D.; "Demonstration of Morphing Technology through Ground and Wind Tunnel Tests;" 48 $8^{\text {th }}$ AIAA Structures, Structural Dynamics, and Materials Conference, Honolulu, HI, April 2007, AIAA 2007-2235.

${ }^{55}$ Kvaternik, R. G.; Juang, J.; Bennett, R. L.; "Exploratory Studies in Generalized Predictive Control for Active Aeroelastic Control of Tiltrotor Aircraft;" NASA Langley Research Center, Hampton, VA; TM-2000-210552.

${ }^{56}$ Kvaternik, R. G.; Piatak, D. J.; Nixon, M. W.; Langston, C. W.; Singleton, J. D.; Bennett, R. L.; Brown, R. K.; “An Experimental Evaluation of Generalized Predictive Control for Tiltrotor Aeroelastic Stability Augmentation in Airplane Mode of Flight;" American Helicopter Society, $57^{\text {th }}$ Annual Forum, Washington, D.C.; May 2001; published July 2002.

${ }^{57}$ Kvaternik, R. G.; Eure, K. W.; Juang, J.; "Exploratory Studies in Generalized Predictive Control for Active Gust Load Alleviation;" NASA Langley Research Center, Hampton, VA; TM-2006-214296.

${ }^{58}$ Silva, W. A.; Vartio, E.; Shimko, A.; Kvaternik, R. G.; Eure, K. W.; Scott, R. C.; "Development of Aeroservoelastic Analytical Models and Gust Load Alleviation Control Laws of a SensorCraft Wind-Tunnel Model Using Measured Data;" 47 AIAA Structures, Structural Dynamics, and Materials Conference, Newport, RI, May 2006, AIAA 2006-1935. 


\section{Appendix A. Aeroelastic Scaling Example, Representative Transport Aircraft}

There are three primary methods of constructing a dynamically-scaled wind tunnel model. Method 1 requires the machining of an aluminum billet (or other metal) to create a tailored thickness distribution of the wing structure. In this design, the majority of material will form a tapered solid beam about the elastic axis with thin plate throughout the rest of the chord. The aerodynamic shape is achieved by bonding either end-grain balsa wood or various types of foam to the plate. Method 2 is a spar-pod design that involves the construction of a primary beam, or wing spar, with segmented aerodynamic pods attached to the beam and distributed masses to achieve the proper mode shapes and mass distribution. Method 3 is a stressed-skin design that typically requires the construction of molds and carefully tailored skins with honeycomb or foam cores.

Method 3 offers the best ability to achieve high structural efficiency. However, it is the most difficult to analyze and construct, it is the most costly option, and it offers the least opportunity for structural tuning or adjustment after construction. It also may have limitations regarding minimum skin gage limiting its efficiency, or the ability to install instrumentation. Method 2 offers the most amount of adjustment after construction, however, is also the least structurally efficient and produces questionable aerodynamics due to the segmented fairings. Therefore, this scaling example will focus on Method 1.

This scaling example is based upon a wing that is representative of a transport aircraft that cruises at Mach 0.84 at an altitude of 30,000 ft. The properties of the representative full-scale wing are: semi-span $=100 \mathrm{ft}$, weight $=$ $105,000 \mathrm{lbs}$, root chord $=42 \mathrm{ft}, \mathrm{MAC}=23 \mathrm{ft}$, first bending frequency $=1.6 \mathrm{~Hz}$, airfoil thickness at the spar location $=12 \%$. The wing weight does not include engine nacelles but does include fuel at a nominal cruise condition. For purposes of this scaling exercise, engine nacelles are neglected so that one can focus upon the ability to model a wing structure.

Figure A1 is an axonometric illustration of the primary wing structure for a wind tunnel model utilizing method 1 construction, shown from the point of view of the wing root. The minimum plate thickness is limited by material warping during machining, and for models sized for TDT is limited to about $1 / 4$ inch in thickness for aluminum. It is assumed that this minimum required plate thickness will scale relative to the size of the model if designed for other facilities.

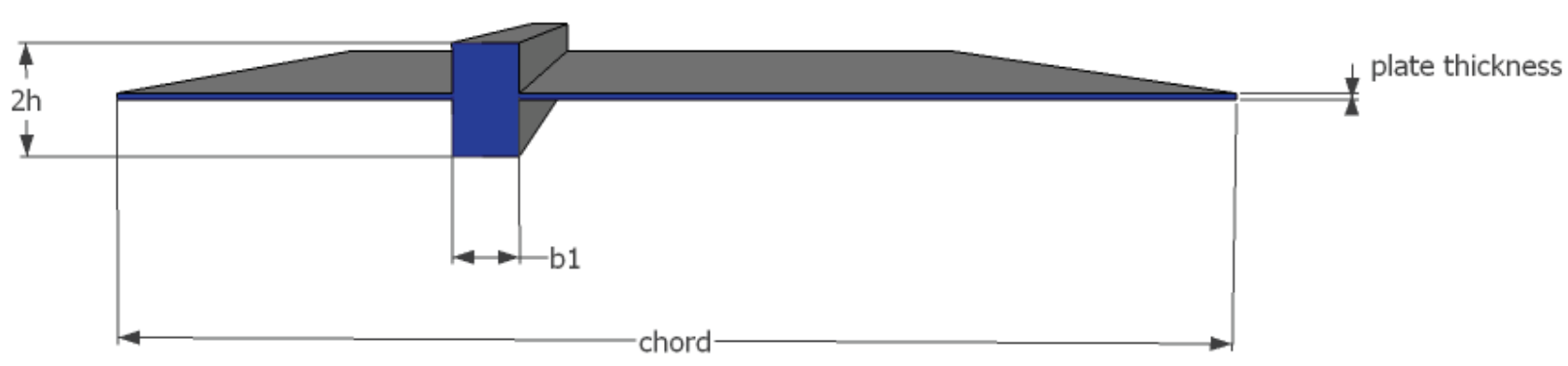

Figure A1. Axonometric projection of wing structure for method 1 construction of wind tunnel model, aerodynamic foam not shown.

Mass and dynamic properties are typically determined by detailed FEM analysis that will then allow the design of tailored thickness distributions. For purposes of this scaling example, a simplified analysis method is required. For initial sizing, a reasonably accurate approach is to treat the wing structure as a beam with the plate and aerodynamic foam contributing only mass and not stiffness. Ref. 6 describes methods for calculating the mode shapes and frequencies of tapered cantilever beams. Example 4-1 of Ref. 6 utilizes these techniques for a tapered cantilever beam with the dimensions as shown in Fig. A2. Utilizing the results of the Modified Raleigh-Ritz method with two polynomials, ${ }^{6}$ the first bending frequency of the beam can be determined by Eq. (A1) for constant width, b1. It is important to note that the frequency is independent of the width of the beam. The independence of frequency upon width can also be shown for uniform beams using the equations from Ref. 26 and decomposing the area moment of inertia into terms of beam height and width.

Appendix A, page A-1

American Institute of Aeronautics and Astronautics 


$$
\omega=2.4769 \sqrt{\frac{E h^{2}}{\rho_{\text {avg }} L^{4}}}
$$

where, $\rho_{\text {avg }}$ is typically $=2.7 \rho_{s}$.

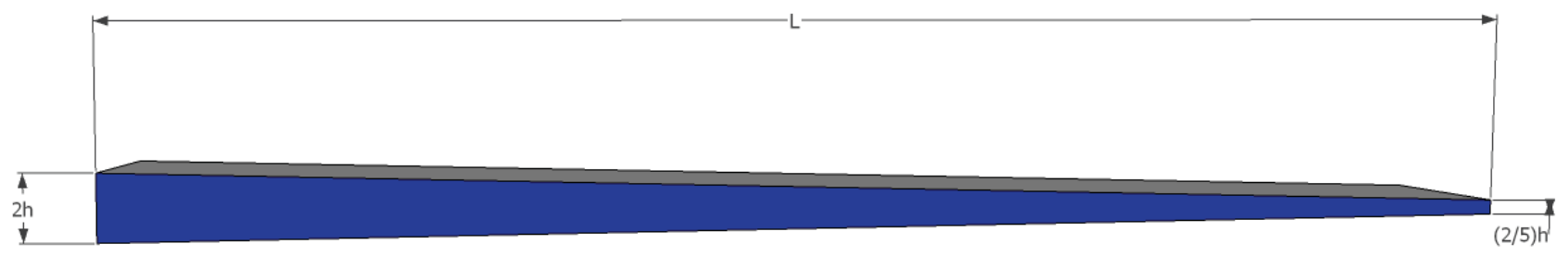

Figure A2. Tapered cantilever beam of Example 4-1 in Ref. 6.

To utilize Eq. A1, the value of $\rho_{\text {avg }}$ must include non-structural weight such as the plate, foam, and ballast. Ballast weights, or any non-structural weight, are required in order to match the mass distribution, mode shapes, higher mode frequencies, and radius of gyration of the full-scale vehicle. It is optimistic, although reasonable, to estimate that $20 \%$ of the model wing weight is due to ballast.

Selecting aluminum for the primary structure of the wind tunnel model, 10-pound foam (10 pounds per $\left.\mathrm{ft}^{3}\right)$, for the aerodynamic fairing, and assuming a $20 \%$ ballast weight increase; $\rho_{\text {avg }}$ will be increased by a factor of 2.7 above $\rho_{s}$ in Eq. (A1). This factor of 2.7 represents the ratio of total wing weight to beam weight, and is calculated in Table A2. Using Eq. (A1), one can now derive the required beam height to achieve the required model-scale frequency, with the limitation being that the required height cannot exceed the wing thickness. Table A1 shows the required model values of a Method 1 design for TDT in R-134a, S1MA in air, S2MA in air, and NTF in cryogenic $\mathrm{N}_{2}$ at $250^{\circ} \mathrm{F}$. As shown in Table A1, the required beam height for S1MA, S2MA, and NTF exceeds the wing thickness. Therefore, a method 1 construction technique cannot be used for these wind tunnels unless the beam uses a material with a significantly higher specific modulus than used for the full-scale aircraft structure. Construction techniques closer to method 3 or some form of tapered hollow beams may allow increased structural efficiency, however, these techniques are significantly more difficult to analyze, construct, and alter after construction.

Table A2 continues the design analysis to determine the model wing weight. Once the model weight is determined, the required flow density can be determined by Eq. (9). In a variable pressure wind tunnel, required flow densities are reached by adjusting tunnel pressure which is related by the equation of state. The italicized and underlined values in Table A2 are those that violate the method 1 construction technique as determined in Table A1. It is assumed that a more sophisticated construction technique is being employed for the models used in S1MA, S2MA, and NTF such that the required stiffness is met without increasing the weight. Making this assumption, the required tunnel pressure of S1MA is greater than four atmospheres. Since S1MA is vented and not variable pressure, S2MA, an aeroelastic test facility, is also considered. The required dynamic pressure is calculated as shown in Eq. (A2) using standard atmospheric values for density and speed of sound at an altitude of 30,000 ft. The remaining values in Table A2 are calculated with Eqs. (A3-A6) and Eqs. $(13,14)$.

$$
\begin{gathered}
q=\frac{1}{2} \lambda_{\rho} \rho_{30,000 f t}\left(M a_{30,000 f t} \lambda_{a}\right)^{2} \\
\lambda_{m}=\frac{\lambda_{\text {weight }}}{\lambda_{L}} \\
\omega=\beta^{2} \sqrt{\frac{E I}{m L^{4}}} \quad \text { from Ref. 26 }
\end{gathered}
$$

where $\beta$ is a scalar that is a function of beam design and mode number. ${ }^{26}$

Appendix A, page A-2

American Institute of Aeronautics and Astronautics 
Rearranging Eq. (A4) results in:

$$
\begin{gathered}
I=\frac{\omega^{2} m L^{4}}{\beta^{4} E} \\
\lambda_{I}=\frac{\lambda_{\omega}{ }^{2} \lambda_{m} \lambda_{L}{ }^{4}}{\lambda_{E}}
\end{gathered}
$$

Table A1. Method 1 Design of Dynamically-Scaled, Mach-Scaled Wind Tunnel Model

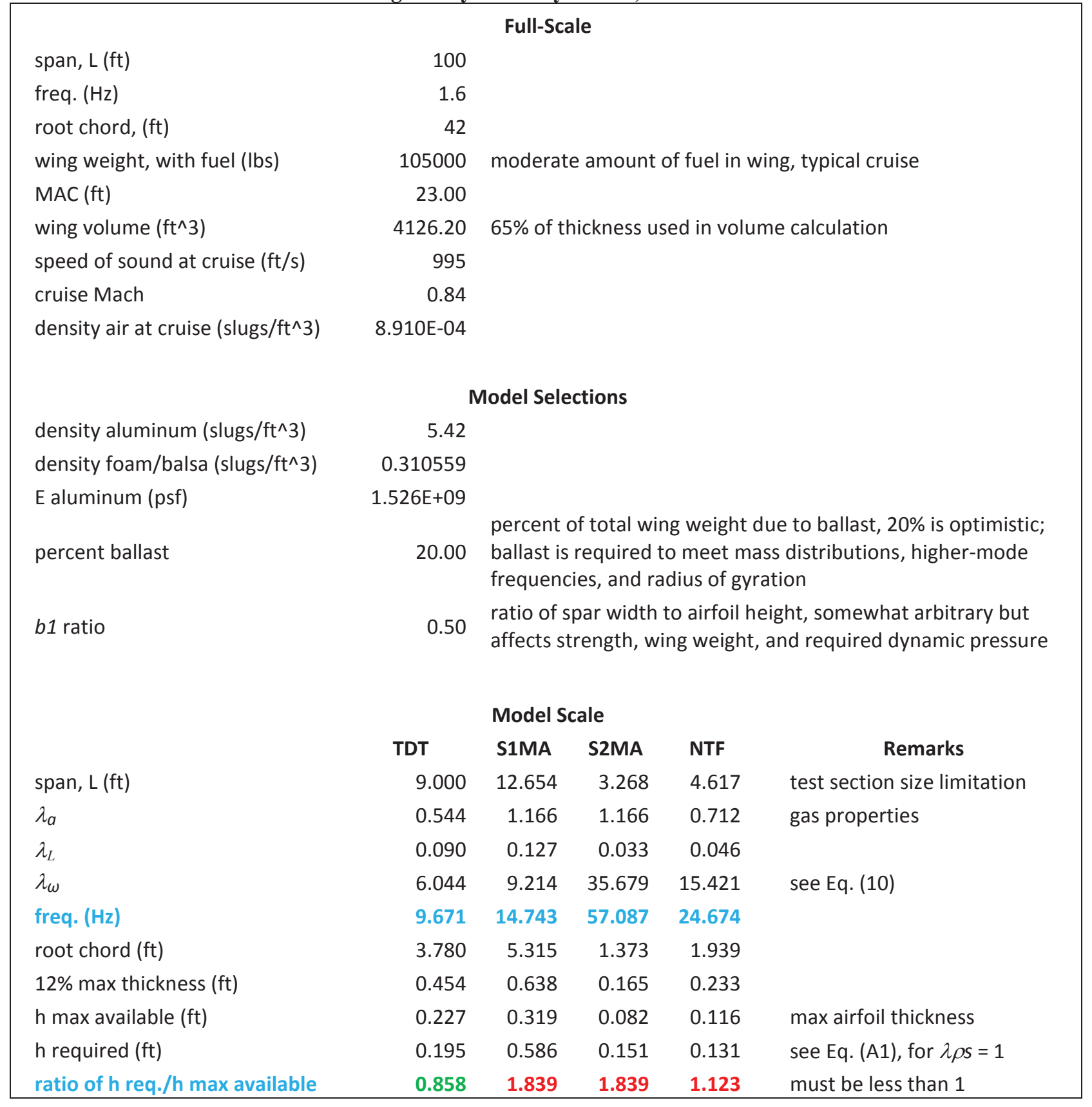


Table A2. Design of Dynamically-Scaled, Mach-Scaled Wind Tunnel Model; Italicized underlined text indicates values violate Method 1 design and utilize more sophisticated design techniques

\begin{tabular}{|c|c|c|c|c|c|}
\hline & TDT & S1MA & S2MA & NTF & Remarks \\
\hline model wing volume $\left(\mathrm{ft}^{\wedge} 3\right)$ & 3.008 & 8.361 & 0.144 & 0.406 & $\lambda_{L}^{3}$ \\
\hline $\mathrm{b} 1(\mathrm{ft})$ & 0.227 & 0.319 & 0.082 & 0.116 & \\
\hline wing beam weight (lbs) & 83.188 & $\underline{495.581}$ & $\underline{8.536}$ & $\underline{14.699}$ & \\
\hline min plate thickness (ft) & 0.021 & $\underline{0.029}$ & $\underline{0.011}$ & $\underline{0.015}$ & $\begin{array}{l}\text { min plate thickness to } \\
\text { prevent warping }\end{array}$ \\
\hline aluminum volume $\left(\mathrm{ft}^{\wedge} 3\right)$ & 0.902 & $\underline{4.022}$ & $\underline{0.078}$ & $\underline{0.165}$ & \\
\hline foam volume $\left(\mathrm{ft}^{\wedge} 3\right)$ & 2.106 & $\underline{4.339}$ & $\underline{0.066}$ & $\underline{0.241}$ & $\begin{array}{l}\text { wing volume - aluminum } \\
\text { volume }\end{array}$ \\
\hline structure weight (lbs) & 178.47 & $\underline{745.25}$ & $\underline{14.20}$ & $\underline{31.20}$ & Includes plate and foam \\
\hline wing weight (lbs) & 223.08 & $\underline{931.56}$ & $\underline{17.75}$ & $\underline{39.00}$ & add ballast weight \\
\hline $\begin{array}{l}\text { ratio of wing weight to } \\
\text { beam weight }\end{array}$ & $2.682 *$ & 1.880 & $\underline{2.079}$ & $\underline{2.653}$ & \\
\hline$\lambda_{\text {weight }}$ & 0.00212 & $\underline{0.00887}$ & $\underline{0.00017}$ & $\underline{0.00037}$ & \\
\hline$\lambda_{\rho}$ & 2.914 & $\underline{4.379}$ & $\underline{4.843}$ & $\underline{3.774}$ & see Eq. (9) \\
\hline $\begin{array}{l}\text { gas density ratio wrt. air } \\
\text { (gas prop.) }\end{array}$ & 3.500 & 1.000 & 1.000 & $\underline{1.000}$ & gas properties \\
\hline $\begin{array}{l}\text { tunnel pressure } \\
\text { (atmospheres) }\end{array}$ & 0.833 & $\underline{4.379}$ & $\underline{4.843}$ & $\underline{3.774}$ & $\begin{array}{l}\text { equation of state, and gas } \\
\text { density ratio }\end{array}$ \\
\hline $\begin{array}{l}\text { dynamic pressure } \\
\left(\mathrm{lbs} / \mathrm{ft}^{\wedge} 2\right)\end{array}$ & 268.4 & $\underline{1852.6}$ & $\underline{2048.9}$ & $\underline{595.4}$ & see Eq. (A2) \\
\hline$\lambda m$ & 0.02361 & $\underline{0.07011}$ & $\underline{0.00517}$ & $\underline{0.00804}$ & see Eq. (A3) \\
\hline$\lambda_{I}$ & $5.66 \mathrm{E}-05$ & $\underline{1.53 E-03}$ & $\underline{7.51 E-06}$ & $\underline{8.69 E-06}$ & see Eq. (A6) \\
\hline$\lambda_{G E O}$ & 0.862 & $\underline{5.953}$ & $\underline{6.584}$ & $\underline{1.913}$ & see Eq. (13) \\
\hline$\lambda_{B A L L A S T}$ & 2.914 & $\underline{4.379}$ & $\underline{4.843}$ & $\underline{3.774}$ & see Eq. (14), for $\lambda \rho s=1$ \\
\hline$\lambda_{B A L L A S T} / \lambda_{G E O}$ & 3.379 & $\underline{0.736}$ & $\underline{0.736}$ & 1.973 & \\
\hline
\end{tabular}

*Weight increase factor used to determine $\rho_{\text {avg }}$ in Eq. (A1) using method 1 design.

Consistent with the findings presented in Table 3 and the discussions of the corresponding section, the ratio of $h_{\text {required }}$ to $h_{\text {available }}$ is independent of length scale. This can be seen in Eq. A1 through dimensional analysis and in Table A1 since the ratio of $h_{\text {required }}$ to $h_{\text {available }}$ is the same for both S1MA and S2MA. This also indicates that a scaling exercise similar to the one in this Appendix will produce similar results for any full-scale aircraft of similar construction, regardless of size.

Important to notice are the dimensional values in Tables A1 and A2 for model frequency, wing weight, and dynamic pressure. The dynamically-scaled model in TDT will have a first bending frequency of $9.671 \mathrm{~Hz}$, will weigh $223 \mathrm{lbs}$, and will have to withstand a dynamic pressure of 268.4 psf. If sophisticated construction techniques or materials are used to construct a dynamically-scaled model for S2MA using the same assumptions elsewhere; it will have a first bending frequency of $57.087 \mathrm{~Hz}$, will weigh only $17.746 \mathrm{lbs}$ and will have to withstand a dynamic pressure of 2048.9 psf, which exceeds the limits of the wind tunnel. As demonstrated in Eq. (12), material stress is proportional to dynamic pressure. Therefore, the material stress of a model dynamically-scaled for air will be nearly an order of magnitude higher than that of a model dynamically-scaled for R-134a. Finally, as the natural frequency increases, the frequency of dynamic events that occur on the model also increases thereby increasing the test risk. 